NUREG/CR-2552
SAND82-0342 ARENE BY

AN̈

Printed March 1984

HUREG/CR--2552

DE84 010557

\title{
CRAC2 Model Description
}

L. T. Ritchie, D. J. Alpert, R. P. Burke, J. D. Johnson,

R. M. Ostmeyer, D. C. Aldrich, R. M. Blond

Prepared by

Sandia National Laboratories

Albuquerque, New Mexico 87185 and Livermore, Calıfornı 94550

for the United States Department of Energy

under Contract DE-AC04-76DP00789

\section{Prepared fir}

\section{U. S. NUCLEAR REGULATORY COMMISSION}


NOTICE

This report was prepared as an account of work sponsored by an agency of the United States Government Neither the United States Government nor any agency thereof, or any of their employees, makes any warranty, expressed or implied, or assumes any legal liability or responsibility for any third party's use, or the results of such use, of any information, apparatus product or process disclosed in this report, or represents that its use by such third party would not infringe privately owned rights

\section{Avarlable from}

GPO Sales Program

Division of Technical Information and Document Control

U S Nuclear Regulatory Commission

Washington, DC 20555

and

National Technical Information Service

Springfield, Virginia 22161

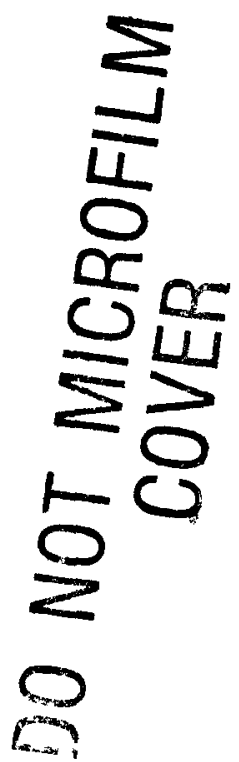




\section{DISCLAIMER}

This report was prepared as an account of work sponsored by an agency of the United States Government. Neither the United States Government nor any agency Thereof, nor any of their employees, makes any warranty, express or implied, or assumes any legal liability or responsibility for the accuracy, completeness, or usefulness of any information, apparatus, product, or process disclosed, or represents that its use would not infringe privately owned rights. Reference herein to any specific commercial product, process, or service by trade name, trademark, manufacturer, or otherwise does not necessarily constitute or imply its endorsement, recommendation, or favoring by the United States Government or any agency thereof. The views and opinions of authors expressed herein do not necessarily state or reflect those of the United States Government or any agency thereof. 


\section{DISCLAIMER}

Portions of this document may be illegible in electronic image products. Images are produced from the best available original document. 
NUREG / CR - 2552

SAND82-0342

AN

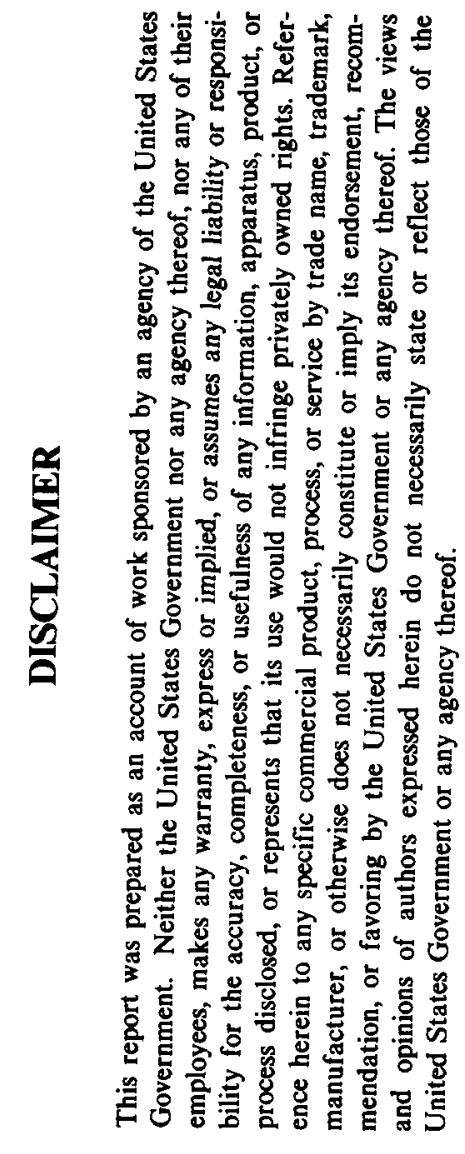

CRAC2

Model Description

L. T. Ritchie

D. J. Alpert

R. P. Burke

J. D. Johnson*

R. M. Ostmeyer

D. C. Aldrich

R. M. Blond**

Date Published: March 1984

Sandia National Laboratories Albuquerque. New Mexico 87185

operated by

Sandia Corporation

for the

U.S. Department of Energy

\author{
Prepared for \\ Division of Risk Analysis \\ Office of Nuclear Regulatory Research \\ U.S. Nuclear Regulatory Commission \\ Washington, D.C. 20555
}

Under Memorandum of Understanding DOE 40-550-75

NRC FIN No. A-1042

* Science Applications, Inc.

** U. S. Nuclear Regulatory Commission 
The CRAC2 computer code is a revised version of CRAC (Calculation of Reactor Accident Consequences) which was developed for the Reactor Safety study. This document provides an overview of the CRAC2 code and a description of each of the models used. Significant improvements incorporated into CRAC2 include an improved weather sequence sampling technique, a new evacuation model, and new output capabilities. In addition. refinements have been made to the atmospheric transport and deposition model. Details of the modeling differences between CRAC2 and CRAC are emphasized in the model descriptions. 
Table of Contents

Chapter

Page

1. Introduction

$1-1$

2. CRAC2 Code Overview

$2-1$

3. CRAC2 Model Desgriptions

$3-1$

3,1 Weather sequence Sampling Method

$3-2$

3.2 The Atmospheric Dispersion Model

$3-8$

3.2,1 Radioactive Decay

$3-11$

3.2.2 Duration of Release

$3-11$

3.2.3 Mixing Layer Depth

$3-11$

3.2.4 Dry and Wet Removal Pracesses

$3-12$

3.2.5 Bujlding Wake Effects

$3-13$

3.2.6 Plume Rise

$3-13$

3.3 Dosimetry Model

$3-15$

3.3,1 Early Exposure

$3+15$

3.3.1.1 Cloudshine

$3-15$

3.3.1.2 Groundshine

$3-16$

3.3.1.3 Inhalation

$3-16$

3.3.2 Chronic Exposure

$3-17$

3.3.2.1 Long-Term Groundshine

3-17

3.3.2.2 Inhalation of Resuspended

$3-19$

3.3.2.3 Ingestion of Radionuclides

$3-20$ from Directly Contaminated Crops

3.3.2.4 Ingestion of Radionuclides 3-22 Via Root Uptake

3.4 Emergency Response Model

$3-26$

3.4.1 Evacuation

$3-26$

3.4 .1 .1

Reactor Safety study

$3-26$

$3.4 \cdot 1$. Evacuation Model

Revised Evacuation Model

$3-27$ 


\section{Page}

3.4.2 Population Sheltering $\quad 328$

3.4.3 Population Relocation 3.29

3.5 Health Effects Model $3-30$

3.5.1 Early Health Effects 3.30

3.5.1.1 Early Fatalities 3.30

3.5.1.2 Early Injuries 3.31

3.5.2 Latent Health Effects 3. 31

3.5.2.1 Latent Cancer Fatalities 3-31

3.5.2.1.1 Latent Cancer 3-32

Fatalities

Resulting from

Early Exposure

3.5.2.1.2 Latent Cancer 333

Fatalities

Resulting from

Chronic Exposure

3.5.2.2 Thyroid Effects 3.34

3.5.2.3 Genetic Effects 336

$\begin{array}{ll}3.6 & \text { Economic Effects Mudel }\end{array} 39$

3.6.1 Costs of Acute Mitigative Actions 3.39

- Evacuation

3.6.2 Costs of Long-Term Mitigative 339

Actions

3.6.2.1 Land Area Interdiction 3.40

Followed by Land and

Property Decontamination

3.6.2.2 Immediate Decontamination 343

and Inhabitation

3.6.2.3 Farm Product Disposal 3.43

3.6.2.4 Crop Disposal 344

3.6 .2 .5 Milk Disposal 344

3.6.2.6 No Long-Term Actions 3.45 
A. The CRAC2 Computer code A-1

A, 1 Concept of operation A-2

A. 2 Program Flow A-5

A.2.1 Program MAIN. THE CRAC2 Code Controller A-5

A.2.2 Subroutine INPUT, the Input Controller $\Lambda-5$

A.2.3 Subroutine SITE. the Accident Site Data Processor

A.2.4 Subroutine BINMET, the Meteorological

A-6

Data sorter

A.2.5 Subroutine RANBIN, the Latin Hypercube

A-6

Initializer

$A-8$

A.2.6 Subroutine SPADAT, the Routine to set spatial Meteorology

A.2.7 Subroutine TIMES, the Generator of stratified Random Times

A.2,8 Subroutine RANDU, the Random Number Generator

A.2.9 Subroutine TIMES2, the Generator of

Random Times

A.2.10 Subroutine EXTRCT, the Meteorological

Data Extractor

A.2.11 Subroutine INCTIM, the Time Incrementing Routine

A.2.12 Subroutine DISP, the CRAC2 Dispersion Model

A.2.13 Subroutine ACTIVE, the CRAC2 Activity Model

A.2.14 Health Effects and Property Damage Routines

$A-8$

A-8

A-8

A-8

A- 8 standard Results

A.2.16 Subroutine STOROPT, the Routine to Process special Results

A-9

A-9

A-9

A -10

A- 16

A-16

B. Quick Reference to the CRAC2 Models

B-1 


\section{List of Figures}

Figure

$\underline{\text { Page }}$

2.1 Schematic Outline of the CRAC2 Code 2-1

3.2-1 Comparison of the Gaussian Distribution 3-10 and Top-Hat Cross Plume Concentration

Distribution Used in CRAC2 and CRAC

A. 1 Spatial Interval Representation

A-2 Flow of MAIN, the CRAC2 Control Program A 17

A. 3 Flow of DISP, the Dispersion Model A-18

A 4 Flow of ACTIVE, the Activity Model A. 19

A 5 Flow of DAMAGE, the Damage Model A 20

A-6 Flow of EARLY, the Early Effects Model A 21

A. 7 Graphic View of the Uniform Dose Interval A 22 Model

A. 8 Flow of the CHRONX, the Chronic Effects Model A. 23

A. 9 Flow of PROPDAM, the Property Damage Model A. 24 


\section{List of Tables}

Table

Page

3.1-1 Weather Category Descriptions 3-6

3.1-2 One Year of New York City Meteorological 3-7

Data Summarized Using the Weather Bin

Categories

3.3-1 Early Time Periods Used to Evaluate Acute Internal Dose to organs.

3.3-2 Nuclides Involved in Chronic Exposure

Calculations

3.5-1 Data Used by CRAC2 to Calculate Latent

Cancer Fatalities

3.5-2 Data Used to Calculate the Incidence of Genetic Disorders and spontaneous Abortions

A-1 Description of Input Subroutines A-7

B-1 Models Adopted for Use in the CRAC2 code B-2

B-2 Importance Sampling of Meteorological Data B-5

B-3 Atmospheric Dispersion Parameterization and B-6 Parameter Values for CRAC2

B-4 Dose-Effect Relationships Used in CRAC2

B-7

Health Effects Models 
Foreword

CRAC2 is a revised version of the CRAC (Calculation of Reactor Accident Consequences) code developed in support of the Reactor safety study [1]. The need for more realistic consequence estimation techniques to be used for such purposes as site evaluation, emergency planning and response, and general risk assessment has guided the development of CRAC2. Sandia National Laboratories has corrected known errors and has made additional changes to the CRAC code. These changes include the introduction of (1) an improved weather sequence sampling technique, (2) a new evacuation model. and (3) new output capabilities: and modification of the (1) atmospheric dispersion model. (2) plume rise model. (3) precipitation scavenging model, and (4) mixing height model. In addition, data utilized by some of the models have been upgraded: (1) latent cancer fatality risk factors have been changed to reflect the lifetime risk of latent cancer from radiation exposure: and (2) economic data have been upgraded to reflect 1980 economic statistics for the United states.

The CRAC2 code has retained the organization, structure, and analytical capabilities of CRAC. but the added capabilities of the CRAC2 code dictated an updated set of documentation to guide its application and use. This model description is one of two documents published to facilitate the informed and intelligent use of CRAC2 and is intended as a detailed description of CRAC2 and its models. It includes an overview of the CRAC2 code and descriptions of each model of the CRAC2 code. with emphasis on the details of the modeling changes that were made in CRAC to produce CRAC2. A guide to the use of the CRAC2 code can be found in the CRAC2 User's Guide [2].

\section{References}

1. WASH-1400 (1975), Reactor Safety Study, Appendix VI: Calculation of Reactor Accident Consequences. NUREG 75/014. US Nuclear Regulatory Commission.

2. Ritchie, L. T., Johnson, J. D. and Blond, R. M. (1983). Calculations of Reactor Accident Accident Consequences, Version 2, CRAC2, Computer Code User's Guide. NUREG/CR-2326, SAND 81-1994. Sandia National Laboratories. 


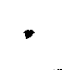




\section{Introduction}

The Reactor safety study was the first comprehensive assessment of the consequences and risks to society from potential accidents at nuclear power plants. As a part of that study. the CRAC computer code was developed to estimate the public consequences of releases of radioactive material to the atmosphere. More specifically. CRAC was designed to assess the aggregate societal risk from potential accidents at generic reactor sites. The application of CRAC to an increasing variety of problems prompted improvements in its modeling capabilities. The CRAC2 code grew out of this desire to provide an improved, more realistic consequence model that was better suited to these applications. CRAC2 was designed as a revision of CRAC, retaining the organization. structure, and analytical capabilities of CRAC, but enhanced by refinements to some of the original models and introduction of some new models and modeling techniques.

This document describes the CRAC2 code and its relationship to CRAC. Because CRAC2 is a modification of CRAC and many models found in the two codes correspond identically. documentation on CRAC will frequently be referred to. In particular. frequent references will be made to Appendix VI of the Reactor Safety study [1] where the CRAC models were originally described.

An overview of the CRAC2 code is given in section 2 . Section 3 consists of descriptions of each model of the CRAC2 code. with particular emphasis placed on the changes that distinguish CRAC2 from CRAC. Appendix A describes the implementation of the CRAC2 models within the CRAC2 computer code, and contains a description of the purpose and flow of each CRAC2 subroutine. Appendix $B$ contains a tabular description of the models utilized in CRAC2 and serves as a quick reference for these models.

\section{References for section 1}

1. WASH-1400 (1975), Reactor Safety Study, Appendix VI: Calculation of Reactor Accident Consequences. NUREG 75/014. US Nuclear Regulatory Commission. 


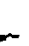




\section{CRAC2 Code Overview}

The CRAC2 code consists of a sequence of mathematical and statistical models which represent the radioactive material immediately after release from containment, the movement of the material as it disperses downwind of the plant. the deposition of the radioactive material onto the ground, and the effects of the airborne and deposited material on man and his environment. The types of consequences calculated by CRAC2 include early and continuing somatic effects. late somatic effects (cancers). genetic effects, and economic impacts. The sequence of models that constitute the CRAC2 code are shown schematically in Figure $2-1$.

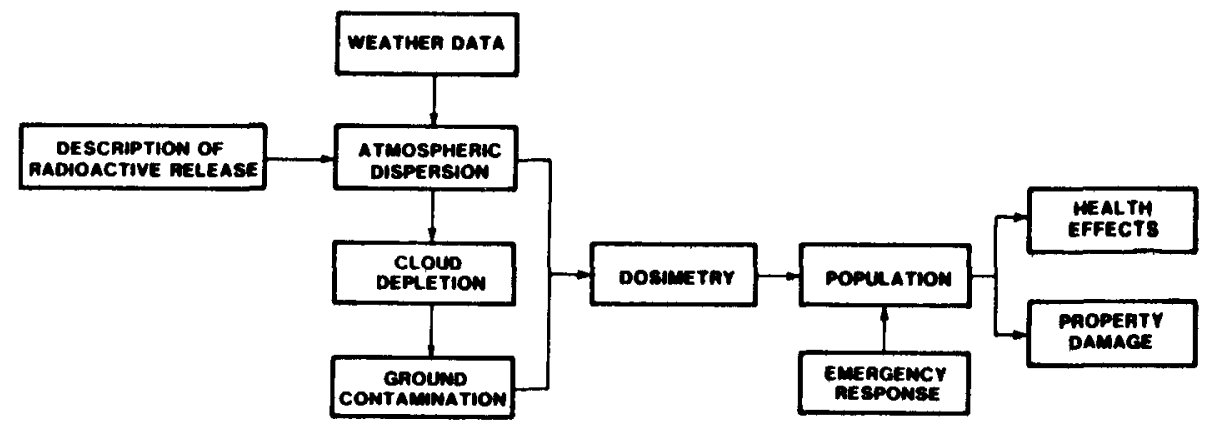

Figure 2-1 schematic outline

of the CRAC2 Model 
Data which must be supplied to the code include a description of the releases of radioactive material (source term) and the meteorological conditions to be considered. The weather conditions during and immediately following the release are treated stochastically. That is. given a source term, the magnitude of consequences are estimated for a variety of meteorological conditions and wind directions, with associated probabilities based on observed meteorological statistics. Thus, the results obtained from the calculations are probabilistic in nature. reflecting the probabilities associated with the accident occurring, the wind direction, and the weather conditions. The distribution of calculated consequences and associated probabilities are presented in the form of complementary cumulative distribution functions (CCDFs). In addition, the expected (or mean) value of the CCDF is calculated.

The first step in a consequence analysis is the calculation of the atmospheric transport. dispersion, and deposition of the released material. To reflect the dependence of the transport and dispersion process on the weather conditions. representative sets of weather sequences are selected from one year of hourly meteorological observations. Several methods are available to select the weather sequences to be analyzed. The goal is to realistically represent the full range of possible weather conditions at a particular site. For each source term and meteorological sequence, the temporally and spatially-dependent airborne concentration and ground contamination are calculated for each radionuclide. The atmospheric transport and dispersion of the plume is modeled with a Gaussian-plume formulation utilizing the well known Pasquill-Gifford parameterization of atmospheric dispersion. The sequence of hourly recordings is used to account for changing weather condition: i.e.. wind speed, atmospheric stability, and precipitation may change during plume passage. The wind direction, however, is assumed to be invariant during and following release and wind shear effects are not considered.

As the plume of radioactive material travels downwind of the reactor. material is removed from the plume and deposited on the ground by both wet and dry deposition. These deposition processes are nuclide dependent. Dry deposition is modeled using a deposition velocity. Wet deposition is modeled using a simple exponential removal rate that is dependent on the rain rate and the thermal stability. In addition to the deposition. radioactive decay. including the buildup of any daughter products. is modeled.

Next. the potential radiation doses that individuals and populations could receive from the released radioactive material are evaluated. The radiation exposures are divided into two categories: early exposure. which occurs during and shortly 
after plume passage, and chronic exposure, which occurs over a longer time period. Three early exposure pathways are considered in CRAC2: 1) direct irradiation by the passing cloud (cloudshine). 2) exposure from inhaled radionuclides, and 3 ) exposure to radioactive material deposited on the ground (groundshine). The chronic exposure pathways include: 1) longterm groundshine. 2) inhalation of resuspended particles, and 3 ) ingestion of contaminated foods. Dose-mitigating actions to reduce both immediate and long-term exposures are included in CRAC2. Measures to reduce immediate exposure include evacuation, sheltering, and relocation. Long-term measures include interdiction of land and food, relocation. and decontamination.

Once the radiation doses to individuals have been evaluated. the magnitude of health effects and economic consequences are calculated. The number of health effects depends on the assumed population distribution. To reflect the impact of wind direction. these calculations are done for each of the specified wind rose directions. The health effects considered include early deaths, early injuries. thyroid effects. latent cancer fatalities, and genetic effects. The offsite economic consequences include costs of interdicting land and crops, emergency response costs, and the cost of decontamination.

In addition to the numbers of each consequence. CRAC2 can produce as output the distribution of doses as a function of time and distance, the largest distance at which certain consequences occur, and the individual risk of certain conseguences as a function of distance. 
3. CRAC2 Model Descriptions

The CRAC2 code consists of the sequence of models shown in Figure 2-1. Six discussion topics have been selected to represent this sequence of models:

1) The Weather sequence Sampling Method:

2) The Atmospheric Dispersion Model:

3) The Dosimetry Model:

4) The Emergency Response Model:

5) The Health Effects Model:

6) The Economic Effects Model.

The following descriptions of the models of the CRAC2 code are in terms of these topics. 


\subsection{Weather sequence Sampling Method}

The atmospheric dispersion of radioactive material from a postulated accident depends on the weather from the start of the accident through a period of tens to hundreds of hours following the accident. The character of the accident together with the weather coincident with and immediately following the accident determines the transport and dispersion process that follows, and thus, the magnitude of the consequences that will result. Since the weather that could occur coincident with the accident is diverse, representative weather data sequences are selected as input to the dispersion model to reflect the dependence of the transport and dispersion process on the site weather. The selection process is done by means of sampling techniques from a full year of hourly weather data characteristic of the plant site. The CRAC2 model allows a choice between four sampling techniques: (1) random sampling: (2) stratified random sampling: (3) stratified sampling: and (4) importance sampling.* Whatever sampling technique is chosen, the goal is to realistically represent the distribution of dispersion model results as a function of the site characteristic weather.

The first three of these sampling methods were included in the original CRAC model. A description of these three methods can be found in section 13 of Appendix VI of the Reactor safety study [1]. The sampling method recommended for use in CRAC, and used in most CRAC applications, is the stratified sampling method. The stratified sampling method ensures a complete coverage of diurnal. seasonal, and four day cycles without the statistical noise of methods that utilize random sampling [1]. Sensitivity studies performed using CRAC indicate considerable variability in predicted results attributable to sampling by this method, however [2]. The importance sampling method available in CRAC2 greatly reduces the variability due to sampling observed with any of these three other techniques.

The basis of the importance sampling method is an initial assessment of the full set of hourly weather data. This initial assessment provides information about the types of weather sequences contained in the data and the frequency of these weather types. With this information, weather sequences can be sampled to reflect the full year's weather data. This ensures representation of each type of weather sequence, those important to realistic representation of the weather data set. and those important to the occurrence of the most serious accident consequences.

\footnotetext{
* The sampling methods described in this section are implemented in subroutines BINMET and RANBIN and parts of MAIN and DAMAGE.
} 
The weather data assessment is done by sorting it into weather categories, categories that provide a realistic representation of the year's weather without overlooking those kinds of weather that are instrumental in producing major consequence impacts. A set of 29 weather categories has been selected for the CRAC2 model to reflect these requirements.

Heuristic judgment played a significant role in the choice of the 29 categories into which the data is sorted. Experience with the CRAC model revealed the impact of weather events on the consequence magnitudes resulting from the accident. Given a postulated large accident. large numbers of early deaths and injuries are normally associated with relatively low probability weather events such as rainfall or wind speed slowdowns within 30 miles of the plant site or with stable weather and moderate wind speeds at the start of the release. In CRAC2 these weather data types have been selected to be among the 29 categories utilized in the assessment process.

The 29 categories are described in Table 3.1-1. An example of weather data sorted into these categories is shown in Table 3.1-2. The weather data for this example represent one year of meteorological data for the City of New York. The entire year of data, 8760 hourly recordings, are sorted into the 29 weather categories. Each sequence is examined to determine (1) the first occurrence of rain within 30 miles of the site. or (2) the first occurrence of a wind speed slowdown within 30 miles of the accident site, or (3) the stability category and wind speed at the start of the sequence. The first of these conditions that is satisfied by the sequence determines the weather category to which it is assigned. Following the assessment process, the start hour of each weather sequence will have been assigned to one and only one weather category. Each of the weather categories then includes a set of weather sequences representing the corresponding weather type. The probability of occurrence of that weather type is the ratio of the total number of weather sequences in the category to the total number of sequences in the year's weather data set.

The sampling procedure now has two key items of information available to it: (1) the category of each weather sequence, and (2) the probability of occurrence of each category of weather. A sample consists of a set of weather sequences selected from each of the categories. Normally. four sequences are selected from each category by the "Latin hypercube" sampling scheme [3]. With this sampling method, random samples are drawn from sets evenly spaced within the weather category. This assures that the model uses an even representation of the weather data over the full year. Assume that a weather category contains $\mathbf{N}_{\mathbf{i}}$ weather sequences and that $K_{i}$ of the sequences are to be selected as samples. $0<\mathrm{K}_{\mathrm{i}} \leq \mathrm{N}_{\mathrm{i}} \cdot$. The $\mathrm{N}_{\mathrm{i}}$ weather sequences are then grouped into $K_{i}$ evenly spaced sets. $s_{1}, \ldots, s_{K_{i}}$. 
set $s_{j}$ contains

$$
\left[j\left(\frac{N_{i}}{k_{i}}\right)\right]-\left[(j-1)\left(\frac{N_{i}}{k_{i}}\right)\right] \text { * }
$$

weather sequences**. One weather sequence is then randomly selected from each set. Since the total number of weather sequences selected from category $i$ would be $K_{i}$. the total number of sequences selected from all 29 of the categories would be

$$
\sum_{i=1}^{29} k_{i}
$$

The assigned probability for a meteorological sequence sampled from category i would be

$$
\frac{N_{i / K_{i}}}{29} \sum_{i=1}^{N_{i}}
$$

\footnotetext{
*The notation

$$
\left[j\left(\frac{N_{i}}{K_{i}}\right)\right]
$$

means the largest integer contained in the number $j\left(\frac{N_{i}}{K_{i}}\right)$.

** Since the $\mathrm{N}_{\mathbf{i}}$ weather sequences of category $\mathrm{i}$ have a natural order determined by the initial time of each of the weather sequences. the evenly spaced sets $s_{1}, \ldots . s_{K_{i}}$ are ordered: i.e... $s_{1}$

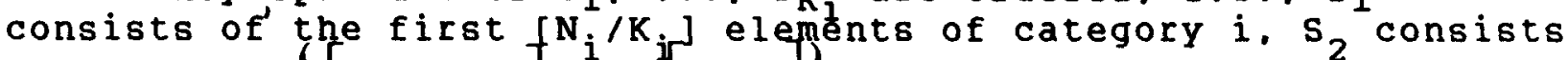
of the next $\left\{\left[2\left(\mathrm{~N}_{\mathrm{i}} / \mathrm{K}_{\mathrm{i}}\right)\right]^{1}-\left[\mathrm{N}_{\mathrm{i}} / \mathrm{K}_{\mathrm{i}}\right]\right\}$ elements of categor $\mathrm{i}$, and so on.
} 
Consider a simple example. Let category i contain 10 weather sequences from which four are to be sampled. Then $\mathbf{N}_{\mathbf{i}}=10$. $K_{i}=4$, and $s_{1}$ contains two sequences. $s_{2}$ contains three sequences. $s_{3}$ contains two sequences, and $\mathrm{s}_{4}$ contains three sequences. One sequence is randomly drawn from each set $s_{j} \cdot$ $\mathrm{j}=1, \ldots 4$, as in the figure below.

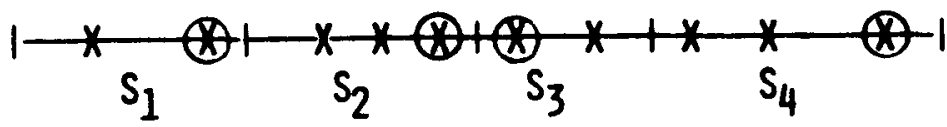

The assigned probability for a sequence chosen from this category would be $\frac{10 / 4}{8760}$. since CRAC2 requires the year's weather data to contain 8760 sequences.

The technique of importance sampling described here selects weather sequences that accurately represent the range of weather sequences in the weather data and their probability of occurrence, and assures selection of sequences that yield severe consequences. The inclusion of these severe accident consequences and of weather sequence probabilities representing each category is key in the realistic representation of the probability distribution function of consequences. The technique is simple and does not require significant additional computation time compared to other sampling methods.

\section{References for Section 3.1}

1. WASH-1400 (1975), Reactor Safety Study, Appendix VI: Calculation of Reactor Accident Consequences. NUREG75/014. US Nuclear Regulatory Commission.

2. Ritchie, L. T. Aldrich, D. C. and Blond, R. M. (1981), "Weather Sequence Sampling for Risk Calculations." Transactions of the American Nuclear Society. Vol. 38.

3. Iman, R. L. and Conover, W. J. (1982), Short Course on Sensitivity Analysis Techniques, NUREG/CR-2350, SANDBI-1978. 
TABLE 3.1-1

Weather Category Descriptions

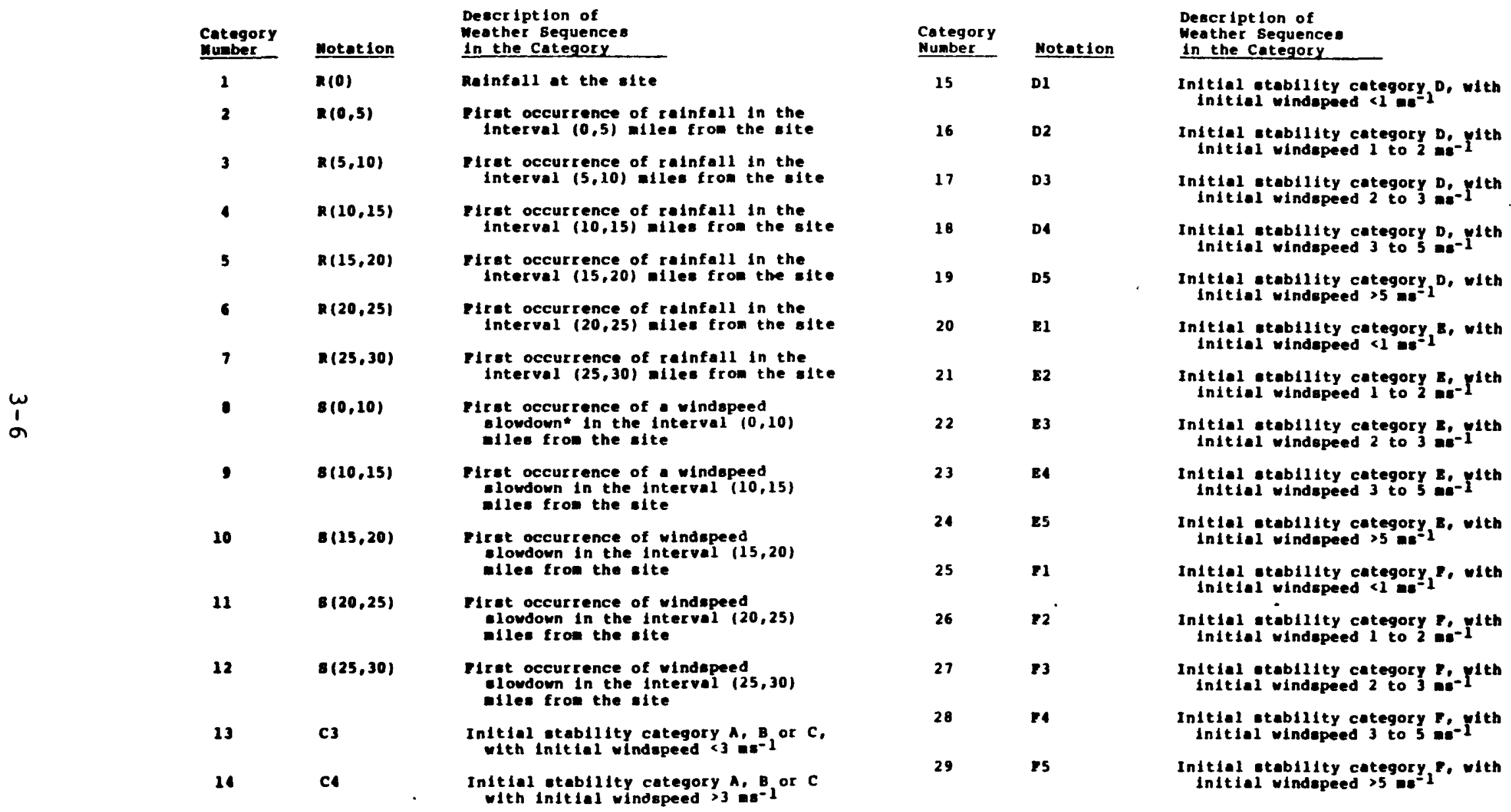

Windgpeed olowdown 18 defined to be windapeed greater than
3 and 
TABLE $3 \cdot 1-2$

One Year of New York City Meteorological Data

Weather Bin Definitions

R - Rain starting within indicated interval (miles).

S - slowdown occurring within indicated interval (miles).

A-C DEF - stability categories.

$1(0-1), 2(1-2), 3(2-3), 4(3-5), 5(G T 5)$ - wind speed intervals $(m / 6)$.

Weather Bin

I R (0)

$2 R(0-5)$

$3 R(5-10)$

$4 R(10-15)$

$5 R(15-20)$

$6 \mathrm{R}(20-25)$

$7 R(25-30)$

8 S $(0-10)$

9 s $(10-15)$

$10 \mathrm{~S}(15-20)$

$115(20-25)$

$12 \mathrm{~S}(25-30)$

$13 \mathrm{C} 3$

$14 \mathrm{C} 4$

$15 \mathrm{D} 1$

$16 \mathrm{D} 2$

17 D 3

$18 \mathrm{D} 4$

$19 \mathrm{D} 5$

$20 \mathrm{E} 1$

21 E 2

22 E 3

23 E 4

24 E 5

25 F 1

$26 \mathrm{~F} 2$

$\begin{array}{lll}27 & F & 3\end{array}$

$28 F$

$29 F 5$
Number of

Sequences

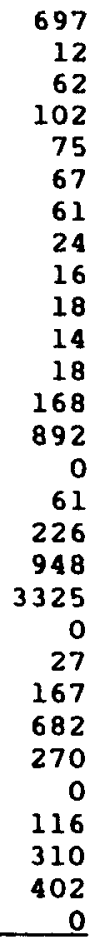

8760
Percent

7.96

.14

.71

1.16

.86

.76

.70

.27

.18

.21

.16

.21

1.92

10.18

0.00

.70

2.58

10.82

37.96

0.00

.31

1.91

7.79

3.08

0.00

1.32

3.54

4.59

0.00

100.00 


\subsection{The Atmospheric Dispersion Model}

The atmospheric transport and dispersion model used in CRAC2 is similar to that used in CRAC [1]. This section will provide a brief outline of the model.* highlighting the differences between CRAC2 and CRAC.

The transport and dispersion model is based on a standard Gaussian-plume formulation [2]. Assuming the material is reflected at the ground, the ground-level, time-integrated concentration for a source of strength $Q$ is given by

$x(x, y, 0)=\frac{\rho}{\pi \sigma_{y}(x) \sigma_{z}(x) \bar{u}} \exp \left(\frac{-y^{2}}{2 \sigma_{y}^{2}(x)}+\frac{-h^{2}}{2 \sigma_{z}^{2}(x)}\right)$

where $\sigma_{y}(x)$ and $\sigma_{z}(x)$, the standard deviations of the crosswind and vertical distributions, respectively, are functions of the downwind distance. $x$. $u$ is the mean wind speed, and $h$ is the source release height. If $Q$ is in curies, the units of $x$ are $\mathrm{Ci}-\mathrm{sec} / \mathrm{m}^{3}$. In CRAC2 and CRAC. equation (i) is simplified by replacing the Gaussian crosswind profile (y direction) with a rectangular (or "top hat") function of width $3 \sigma_{Y}: i . e .$. in equation (1) the term

$$
\frac{1}{\sqrt{2 \pi} \sigma_{Y}(x)} \exp \left(\frac{-y^{2}}{2 \sigma_{Y}^{2}(x)}\right)
$$

is replaced by $1 /\left(3 \sigma_{y}(x)\right)$. With this substitution, equation (1) becomes

$$
x(x, 0)=\frac{Q}{\frac{3}{2} \sqrt{2 \pi} \sigma_{y}(x) \sigma_{z}(x) \bar{u}} \exp \left(\frac{-h^{2}}{2 \sigma_{z}^{2}(x)}\right)
$$

* The models of transport, dispersion, and deposition described in this section are implemented in subroutines SPADAT, DISP and ACTIVE. 
The top-hat function which replaces the Gaussian crosswind profile is shown in Figure 3.2-1. The amplitude of the top hat is 0.836 of the Gaussian peak: however, the area under the top hat curve is identical to the area under the Gaussian crosswind profile.

For each start-hour selected by the meteorological sampling technique, the CRAC2 dispersion model uses the subsequent meteorological conditions to predict the dispersion and transport of the released cloud of radioactive material. The sequence of hourly recordings is used to account for changing weather conditions: i.e.. wind speed. atmospheric stability, and precipitation may change during plume passage. The wind direction. however. is assumed to be invariant during and following the release.

Based on the windspeed in each hour, the stability, windspeed, and accumulated precipitation are assigned to all spatial intervals which the plume passes during the hour. If the windspeed for an hour is not sufficient for the plume to fully traverse an interval. the windspeed. stability and accumulated precipitation are averaged for all hours the plume is within that interval (the average of $A$ and $C$ stability is $B$, of $A$ and $B$ is $B$ ).

Values of $\sigma_{Y}(x)$ and $\sigma_{Z}(x)$ are calculated for each spatial interval using Pasquill-Gifford curves as provided in Turner [2]. The empirical, best-fit functions for the curves given by Martin and Tikvart [3] are used. Successive growth rates of $\sigma_{Y}(x)$ and $\sigma_{z}(x)$ for each spatial interval are estimated from the value in the previous spatial interval by calculating the virtual-source distance at the current stability class necessary to give that value, and extrapolating growth to the end of the current spatial interval. A modification of the vertical dis persion parameters $\left(\sigma_{z}\right)$ for surface roughness was incorporated in CRAC2. The Pasquili-Gifford curves are appropriate for a surface roughness of about $3 \mathrm{~cm}$ (short grass). However, a more typical value for the United states is $10 \mathrm{~cm}$ (crops, bushes. etc). The parameterization of $\sigma_{z}$ incorporated in CRAC2 includes the following surface roughness correction recommended by an AMS workshop [4]:

$$
\sigma_{\mathrm{z}_{\text {CRAC2 }}}={ }^{\sigma} \mathrm{z}_{\mathrm{P}-\mathrm{G}}\left(\frac{10 \mathrm{~cm}}{3 \mathrm{~cm}}\right)^{0.2}
$$

Modifications of equation (3) are incorporated in CRAC2 to account for the effects of: (1) radioactive decay. (2) duration 


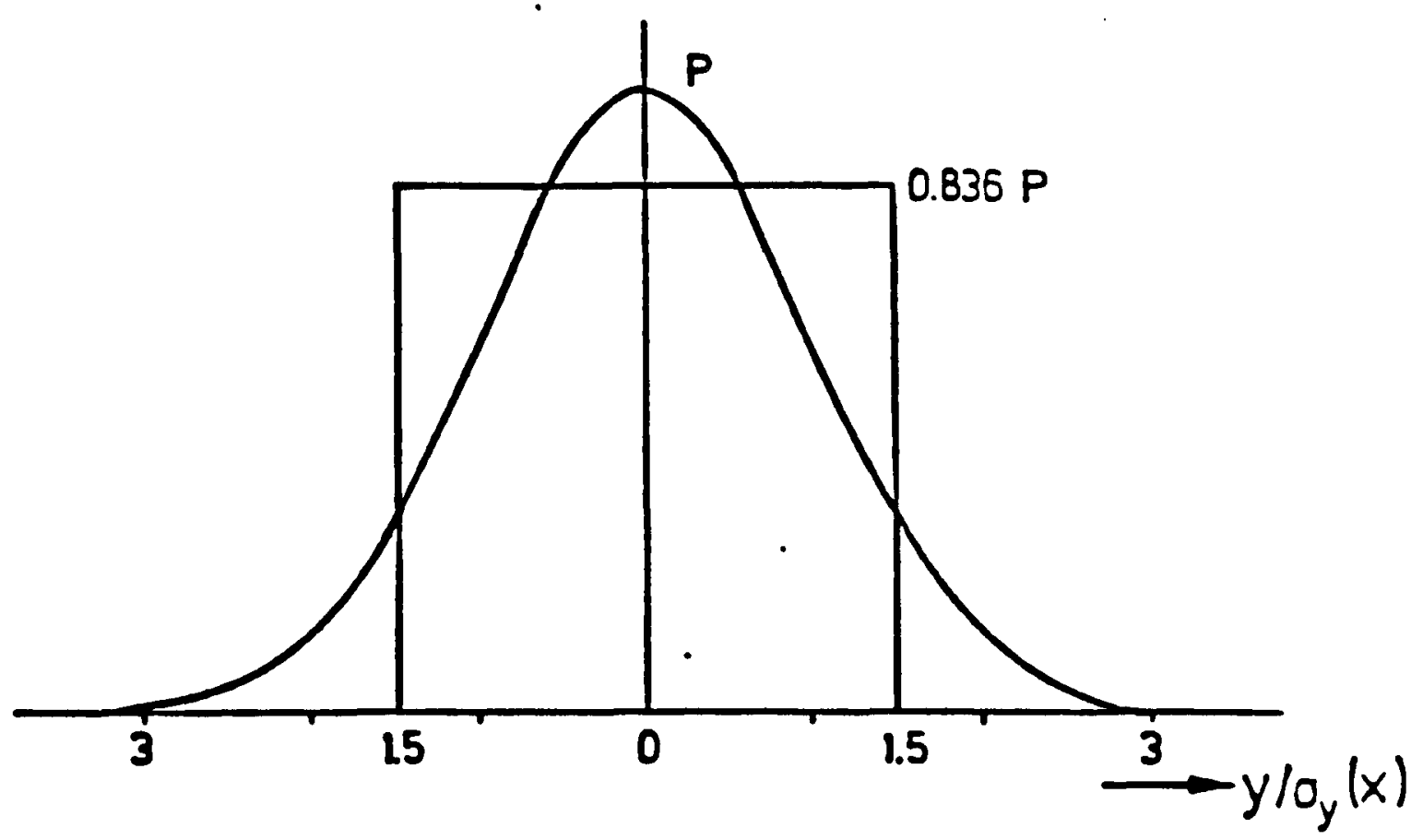

Figure 3.2-1. Comparison of a Gaussian Distribution and the Top-Hat Cross Plume Concentration Distribution Used in CRAC2 and CRAC 
of release, (3) mixing layer depth, (4) dry and wet removal processes. (5) building wake, and (6) plume rise caused by sensible heat buoyancy. The implementation of these modifications is as follows.

\subsubsection{Radioactive Decay}

The radioactive decay of each isotope during downwind transport, including build-up of any daughter products, is evaluated using standard formulae. e.g..

$$
Q(x)=Q \exp (-\lambda x / \bar{u})
$$

where $\lambda$ is the radioactive decay constant.

\subsubsection{Duration of Release}

The standard Pasquill-Gifford curves are appropriate for a release duration of 3 minutes. To account for meander of the plume for releases of longer duration. the horizontal dispersion parameter $\left(\sigma_{y}\right)$ is increased according to the formula recommended by Gifford [5]. For a release duration of $T$ minutes

$$
\sigma_{Y(T)}=\sigma_{Y(3 \text { minutes })}\left(3 \frac{T}{\min }\right)^{Q} \text {. }
$$

where $Q=0.2$ when $3<T \leq 60$ minutes and $Q=0.25$ when $60<T$ $\leq 600$ minutes. Releases of duration greater than 600 minutes (10 hours) are treated as 10 hour releases.

\subsubsection{Mixing Layer Depth}

A limit is imposed on the expansion of $\sigma_{z}$ by the existance of a mixing layer of depth L. For unstable and neutral conditions a seasonal mixing height (included at the end of the meteorological data file) is used. The Pasquili-Gifford expansion is used until $\sigma_{z}$ reaches a value of 0.465 L (at a distance of $\left.x=x_{L}\right)$. Beyond $x_{L}, \sigma_{z}$ expands linearly to a distance of $2 x_{L}$ and $a$ value of $\sigma_{z}\left(2 x_{L}\right)=0.8 \mathrm{~L}$. Further growth of $\sigma_{z}$ is capped at $0.8 \mathrm{~L}$. Limiting the growth of $\sigma_{2}$ to $0.8 \mathrm{~L}$ approximates at ground level the concentration of a uniform vertical profile. For stable conditions (E and F stability), the inversion layer is ground based and no mixing depth is assumed in CRAC2. If, as a result of a change in stability during plume travel, $\sigma_{z}$ exceeds $0.8 \mathrm{~L}, \sigma_{z}$ is held constant. 


\subsubsection{Dry and Wet Removal Processes}

Deposition of each isotope by dry and wet removal is calculated by the source depletion method [6]. The rate that material is deposited on the ground by dry deposition is given by

Flux to Ground $\left(\mathrm{Ci} / \mathrm{m}^{2}-\mathrm{sec}\right)=\left(\begin{array}{l}\text { Air Concentration } \\ \left(\mathrm{Ci} / \mathrm{m}^{3}\right)\end{array}\right)\left(\begin{array}{l}v_{d}(\mathrm{~m} / \mathrm{sec})\end{array}\right)$

where $v_{d}$ is the deposition velocity.

In CRAC2, the fraction of each isotope removed in a spatial interval by dry deposition is approximated by

$$
f_{d}=\frac{v_{d} t_{i}}{h_{e f f}}
$$

where $v_{d}$ is the deposition velocity of the isotope, $t_{i}$ is the time required for the plume front to traverse the interval. and $h_{\text {eff }}$ is the effective plume height:

$h_{\text {eff }} \frac{1}{x(0)} \int_{0}^{\infty} x(z) d z=\left(\frac{\pi}{2}\right)^{1 / 2} \sigma_{z}(x) \exp \left(\frac{h^{2}}{2 \sigma_{z}^{2}(x)}\right)$

CRAC2 incorporates an improved model of wet deposition (washout) which is dependent on rainfall rate. The fraction of each isotope removed by wet removal (washout) is given by

$$
f_{w}=1-\exp \left(-\Lambda t_{i}\right)
$$

$\Lambda$ is the washout coefficient and is defined to be

$$
\Lambda=C R \text {. }
$$

where $R$ is the amount of observed rain in the spatial interval $(\mathrm{mm})$ and $\mathrm{C}$ is $10^{-3}(1 / \mathrm{mm}-\mathrm{sec})$ for unstable and neutral conditions and $10^{-4}(1 / \mathrm{mm}-\mathrm{sec})$ for stable conditions. Alternatively. the original CRAC model which uses a single rain rate $(0.5 \mathrm{~mm} / \mathrm{hr})$ 
may be used. Both $v_{d}$ and $\Lambda$ are generally assumed to be zero for noble gases.

\subsubsection{Building Wake Effects}

To account for the wake effect of a reactor building of height $A$ and width $W$, the initial plume dispersion parameters are initialized to $\sigma_{y}=1 / 3 \mathrm{~W}$ and $\sigma_{z}=1 / 2.15 \mathrm{~A}$. These values correspond to the width of the plume $\left(3 \sigma_{y}\right)$ being equal to the building width and the concentration of the plume at the building top being one-tenth of the centerline value. In addition. if the release height $(h)$ is less than $A, h$ is set equal to zero. A "virtual source" approach is used to calculate the distance to the virtual origin.

\subsubsection{Plume Rise}

In CRAC2 the calculation of buoyant plume rise is made using the updated methods summarized by Briggs [7]. For stable conditions, the following formula is used for the final plume centerline height

$$
h_{\text {final }}=2.6\left(3.7 \times 10^{-5} \mathrm{Q}_{\mathrm{H}} /(\overline{\mathrm{u}} \cdot \mathrm{s})\right)^{1 / 3}+\mathrm{h}
$$

where $Q_{H}$ is the sensible heat release rate (cal/sec), $\bar{u}$ is the average wind speed, and $s$ is a stability parameter

$$
s=\frac{g}{T} \frac{\partial \Theta}{\partial z} \sec ^{-2} \text {. }
$$

there $\mathrm{g}$ is $9.81 \mathrm{~m} / \mathrm{sec}^{2}$. $T$ is the temperature in degrees Kelvin, $\theta$ is the potential temperature in degrees Kelvin, and 2 is the height in meters. The stability parameter $s$ is equal to $8.7 \mathrm{x}$ $10^{-4}$ for $E$ stability and $1.75 \times 10^{-3}$ for $F$ stability. used:

For neutral or unstable conditions, the following relation is

$$
\mathrm{h}_{\mathrm{final}}=1.6\left(3.7 \times 10^{-5} \mathrm{Q}_{\mathrm{H}}\left(\mathrm{x}^{\star^{2}}\right)\right)^{1 / 3} \overline{\mathrm{u}}^{-1}+\mathrm{h} \text {. }
$$

$$
\text { where } x^{\star}=2.08\left(3.7 \times 10^{-5} Q_{H}\right)^{2 / 5}(\mathrm{~h})^{3 / 5} \text {. }
$$


At a distance of $5 x^{*}$ the maximum plume rise is set equal to $58.6 / 25$ of the height at $x^{*}$. The centerline plume rise at the midpoint of each spatial interval is determined by linearly interpolating the height between the origin and $x^{*}$ and between $x^{*}$ and $5 x^{*}$. Generally. the final plume height is reached within about $1 \mathrm{~km}$.

\section{References for Section 3.2}

1. WASH-1400 (1975), Reactor Safety Study, Appendix VI: Calculation of Reactor Accident Consequences. NUREg 75/014. US Nuclear Regulatory Commission.

2. Turner, D. B. (1970), Workbook of Atmospheric Dispersion Estimates. US EPA Office of Air Programs, Publ No 999-AP-26, Research Triangle Park, NC.

3. Martin, D. O. and J. A. Tikvart (1968), "A General Atmospheric Diffusion Model for Estimating the Effects of Air Quality of One or More Sources." presented at the 61st Annual Meeting of the Air Pollution Control Association.

4. "American Meteorological Society Workshop on stability Classification schemes and Sigma Curves--summary and Recommendations" (1977). Bulletin of the American Meteorological Society. Vol 58 .

5. Gifford. F. (1975). "Atmospheric Dispersion Models for Environmental Pollution Applications." in Lectures on Air Pollution and Environmental Impact Analysis. D. A. Haugen. ed AMS. Boston, page 42 .

6. Slade, D. H. (Ed) (1968), Meteorology and Atomic Energy 1968. USAEC TID-24190.

7. Briggs, G. A. (1975), "Plume Rise Predictions," in Lectures on Air Pollution and Environmental Impact Analysis. Workshop Proceedings, American Meteorological Society, Boston, Mass., pp 59-111. 


\subsection{Dosimetry Model}

The calculation of radiation doses in CRAC2 is divided into two categories: early exposure which occurs during and shortly after plume passage, and chronic exposure which occurs over a longer time period. These two classes of exposure are discussed separately. Section 3.3.1 discusses the calculation of doses from early exposure and section 3.3.2 discusses the calculation of doses for chronic exposure.

\subsubsection{Early Exposure}

The early exposure model* in CRAC2 is essentially the same as that in CRAC except for modifications to reflect the new emergency response model implemented in CRAC2 (see section 3.4). The calculation of radiation doses due to early exposure considers three pathways: 1) direct external exposure to radioactive material in the passing cloud (cloudshine), 2) exposure due to the inhalation of radionuclides from the cloud, and 3 ) exposure to radioactive material deposited on the ground (groundshine)[1]. Doses are first calculated for organs specified in the ACUTE subgroup and then for those organs specified in the LATENT subgroup. The radiation doses calculated due to early exposure are strongly dependent on the assumed emergency response, i.e.. evacuation, sheltering, or early relocation. Beyond the specified evacuation distance. cloudshine and inhalation exposures are limited to the time of cloud passage. Groundshine exposure is limited to the exposure time specified in the EVACUATE subgroup. The time periods used to calculate the total committed dose for each organ are discussed in section 9.2 of Appendix VI of the Reactor Safety study (RSS). The following sections describe the calculation of radiation exposures for each of the three pathways.

\subsubsection{Cloudshine}

Estimates of doses due to external exposure to the passing cloud incorporate a "semi-infinite cloud" approximation (see section 8.2 of RSS). First, the time-integrated air concentration. Ac $\left(\mathrm{Ci}-\mathrm{sec} / \mathrm{m}^{3}\right)$, is obtained by multiplying the calculated air concentration by the duration of the exposure (exposure duration is modified by the emergency response). Second, the dose due to immersion in a semi-infinite cloud. Ec (rem), is calculated by multiplying $A C$ by the semi-infinite cloud dose conversion factor. $\mathrm{DC}_{\infty}\left(\mathrm{rem} / \mathrm{Ci}-\mathrm{sec} / \mathrm{m}^{3}\right)$, for each organ and

*The early exposure model described in this section is implemented in subroutine EARLY and part of the EVACU subroutine. 
nuclide.

$$
E C=A C \cdot D C_{\infty}
$$

The dose conversion factors used in CRAC2 are the same as used in CRAC and were calculated for each organ and nuclide using the EXREM III computer code[2] developed at the Oak Ridge National Laboratory. The factors for the total bone marrow. lung. testes, and the whole body are given in RSS Table VI C-1. Next. the dose is reduced by multiplying by the appropriate shielding factor for direct exposure to the cloud (shielding factors are determined by the emergency response). Finally. a correction factor $\left(D c / D c_{\infty}\right)$ is used to correct the calculated semi-infinite cloud doses for a finite cloud. These correction factors, based upon formulations in slade[3] for a spherical cloud, are dependent on the centerline height and vertical standard deviation of the plume. The correction factors used in CRAC2 are given in RSS Table VI 8-1. An interpolation procedure is used to estimate values not given in the table.

\subsubsection{Groundshine}

Groundshine doses (Eg. rem) due to exposure to radioactive material deposited on the ground by both wet and dry deposition are calculated by multiplying the initial level of ground contamination for each nuclide (GC. Ci/m ${ }^{2}$ ) by the appropriate shielding factor ( $\mathrm{SFg}$ ) and by the dose conversion factor (Dg, $\left.\mathrm{rem} / \mathrm{Ci} / \mathrm{m}^{2}\right)$.

$$
\mathbf{E g}=\mathbf{G C} \cdot \mathrm{Dg} \cdot \mathbf{S F g}
$$

Groundshine doses are computed for 8 hour and 7 day exposure periods. For exposure periods between 8 hours and 7 days. doses are determined by interpolating between these two values. For exposure periods shorter than 8 hours, doses are computed by extrapolation of the 8 hour dose. The appropriate shielding factors are determined by the emergency response. The integrated dose-conversion factors used in CRAC2 for exposure periods of 8 hours and 7 days are given in RSS Table VI C- 1 .

\subsubsection{Inhalation}

The internal radiation dose due to inhalation of radionuclides from the passing cloud is calculated for 11 different time periods following exposure. The 11 time periods are: the early (or acute) exposure period (see Table 3.3-1), 0 to 1 year. 1 to 10 years. 11 to 20 years. 21 to 30 years. 31 to 40 years. 41 to 50 years. 51 to 60 years. 61 to 70 years. 71 to 80 years. and greater than 80 years. For each of these time periods, a 
corresponding radiation dose (dose committment) is calculated due to the internally deposited radionuclides. The doses are calculated using the integrated air concentration of each nuclide ( $\mathrm{AC}$. Ci-sec/m $\mathrm{m}^{3}$ ) adjusted for the duration of exposure (modified by the emergency response). The quantity of radioactive material inhaled is calculated by multiplying Ac by the assumed breathing rate ( $\left.\mathrm{Br}, \mathrm{m}^{3} / \mathrm{sec}\right)$. Finally, the dose from inhaled radionuclides ( $E$ i. rem) is found by multiplying the quantity of inhaled material by the dose conversion factor (Di. rem/Ci inhaled) for each organ and time period. Thus:

$$
\mathrm{E} \mathbf{i}=\mathrm{AC} \cdot \mathrm{Br} \cdot \mathrm{Di}
$$

The dose conversion factors for inhalation exposures used in CRAC2 are described in RSS Appendix VI section 8-4. Assumed in the calculation of the dose factors is a particle aerodynamic mean diameter of 1 micron.

\subsubsection{Chronic Exposure}

The chronic exposure model* used in CRAC2 is identical to the CRAC chronic exposure model described in the RSS[1]. The model is described here in terms of its computer code implementation. Calculations are performed for each spatial interval. The discussion in this section will concentrate on how the calculations are performed for each interval.

The calculation of doses due to chronic exposure considers three pathways: 1) long-term exposure to ground contamination. (groundshine). 2) inhalation of resuspended material and 3 ) ingestion of contaminated food. The nuclides that are considered in each of these pathways are shown in Table 3.3-2. These exposure pathways will be discussed separately below.

\subsubsection{Long-Term Groundshine}

The bases for the long-term groundshine model used in the chronic exposure model are discussed in detail in Appendix VI of the RSS. Before calculating doses to an individual, the model first determines whether protective actions should be taken. such as land decontamination or prohibiting land use. To do this. the code compares the long-term groundshine dose to a dose criterion. i.e.. a dose level that would require protective actions (for CRAC2, this level is generally assumed to be 25 rem in 30 years to the the whole body). This groundshine dose is integrated from the time of the accident.

*The chronic exposure model described in this section is implemented in the subroutine CHRONX and parts of subroutine CHRON. 
If the long-term dose does not exceed this dose criterion. no actions are assumed and groundshine doses are integrated from the time of the accident. If the long-term dose exceeds the criterion. the model determines whether or not decontamination of the area can reduce the groundshine doses to an acceptable level. This is done by comparing the ratio of the unperturbed 30-year groundshine dose and the long-term dose criterion to the maximum decontamination factor (CRAC2 uses a factor of 20.0). If this ratio is less than 20.0. groundshine doses are integrated from the time of the accident. However, these doses are calculated assuming that the area will be decontaminated to a level just sufficient to meet the maximum permissible dose criterion. If the ratio exceeds 20.0 . the long-term dose received after one year of decay is compared to the dose criterion. If the ratio does not exceed 20.0. the long-term groundshine dose is integrated starting one year after the accident. These doses are calculated assuming a decontamination factor (DF) that is just large enough to meet the dose criterion after one year. If the ratio exceeds 20.0, a similar comparison is performed for two years and if the two year ratio exceeds 20.0 . a ratio is determined for groundshine doses after 30 years. If the 30 year ratio exceeds the DF of 20.0 , then the land is assumed to be permanently prohibited from use (i.e.. doses are not calculated for any of the pathways). For cases in which the 30 year ratio is less than 20.0 , and the 2 year ratio is greater than the DF of 20.0, the model determines the time between 2 and 30 years for which weathering, radioactive decay and decontamination together are sufficient to allow reinhabitation of the area. Once this time after the accident has been determined. it is called TIMEK, groundshine doses are calculated for the time period after TIMEK using a $D F=20.0$.

The dose calculations are made using the following generalized equation:

$$
\begin{aligned}
\operatorname{DSCOM}(i, \ell, 1)= & \sum_{j=1}^{10}[\operatorname{PROFAC}(\operatorname{NEXP}) \cdot \operatorname{GRCON}(\ell, 3, j) \cdot G C(j, k) \\
& \cdot\left(a \cdot \exp (-\mathrm{C} \cdot \mathrm{TIMEK}) \cdot\left(\exp (-\mathrm{C} \cdot \mathrm{T})-\exp \left(-c \cdot T^{\prime}\right)\right)+\right. \\
& \left.\left.b \cdot \exp (-d \cdot \operatorname{TIMEK}) \cdot\left(\exp (-d \cdot T)-\exp \left(-d^{\prime} \cdot T^{\prime}\right)\right)\right) / \operatorname{DEC}\right]
\end{aligned}
$$

where

$$
\begin{aligned}
& a=0.63 /(1.13+253 / \operatorname{HALF}(j)), \\
& b=0.33 /(0.0075+253 / \operatorname{HALF}(j)) . \\
& c=1.13+253 / \operatorname{HALF}(j) . \\
& d=0.0075+253 / \operatorname{HALF}(j) .
\end{aligned}
$$

For this case. DSCOM is the individual dose to organ $\ell$ in time interval $i$ with endpoints of $T$ and $T^{\prime}$ (e.g.. the 0 to 1 year 
interval after TIMEK has endpoints of 0 and 1 years). The total dose is the sum of the dose from the 10 nuclides considered in this pathway. PROFAC is used to account for ground roughness and shielding. GRCON is the ground rate dose factor for organ $l$ and radionuclide $j$ with units of $\mathrm{rem} / \mathrm{Ci} / \mathrm{m}^{2} / \mathrm{year}$. GC is the initial ground concentration in interval $k$ for radionuclide $j$. HALF (j) is the half-life of radionuclide $j$ in days, and DEC is the decontamination factor. This equation was determined by integrating the weathering and decay equation given in Appendix VI of the RSS from TIMEK + T to TIMEK + T'. As discussed ear lier. TIMEK can equal 0.0. 1 or 2 years, or some figure between 2 and 30 years. DEC can take values from 1.0 to 20 for TIMEK $=0,1$, or 2 years and will be 20.0 for TIMEK greater than 2 years and less than or equal to 30 years. A similar form of this equation is used to determine the maximum possible ground concentration. The individual dose commitments. DSCOM, are

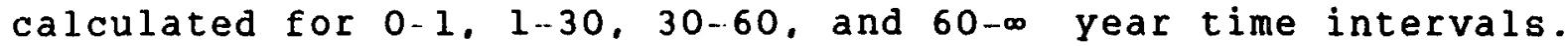
When calculating population doses in an interval, the model assumes a constant population with time.

\subsubsection{Inhalation of Resuspended Radionuclides}

In addition to calculating groundshine doses to individuals residing in the contaminated area, the chronic exposure model calculates doses resulting from inhalation of resuspended radionuclides. These doses are calculated for time intervals after TIMEK, which was determined with the groundshine calculations. Resuspension doses are not calculated if the area is permanenty interdicted.

Exposures received by an individual in time interval $i$ following TIMEK, from radionuclide $j$ in spatial interval $k$, are calculated using the following general equation:

$$
\begin{aligned}
\operatorname{INHAL}(i, j)= & {[\operatorname{GCEOF}(j) \cdot 7300 \cdot G C(j, k)} \\
& \cdot\left(10^{-5} / b\right) \cdot \exp (-b \cdot T I M E K) \cdot\left(\exp (b \cdot T)-\exp \left(-b \cdot T^{\prime}\right)\right)+ \\
& \left.\left.\left(10^{-9} / \lambda\right) \cdot \exp (-\lambda) \cdot T I M E K\right) \cdot\left(\exp (-\lambda \cdot T)-\exp \left(-\lambda \cdot T^{\prime}\right)\right)\right] .
\end{aligned}
$$

where

$$
\begin{aligned}
& b=0.677+253 / \operatorname{HALF}(j) . \\
& \lambda=253 / \operatorname{HALF}(j) .
\end{aligned}
$$

This equation was derived by integrating the resuspension equation given in Appendix VI of the RSS from TIMEK + T to TIMEK + $T^{\prime}$. Variables are as defined for the groundshine exposure pathway. GCEOF is used to account for situations where a radionuclide has a parent. For situations where the nuclide has no 
parent. GCEOF $=1.0$. If a parent is present, GCEOF is modified to account for the additional exposure. Exposures are calculated for the following intervals: 0-10, 10-20, 20-30, 30-40.

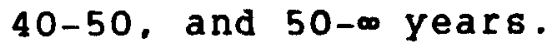

Doses that are received by an individual to organ $\ell$ in the $i$ time intervals after TIMEK are calculated as follows.

$\operatorname{DSCOM}(2, \ell, i)=\sum_{j=1}^{9} \sum_{m=1}^{i} \operatorname{DCINH}(j, \ell, i+1-m) \cdot \operatorname{INHAL}(m) / \operatorname{DEC}$

The total dose is the sum of the doses from the 9 nuclides considered in this pathway. The interval exposures. INHAL, are for the intervals identified above. The DCINH are dose conversion

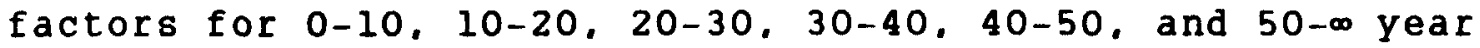
time periods after exposure. The CRAC2 code uses the 40-50 year factor for the 50-- year time period. The decontamination factors. DEC. used for the calculation are those used for the groundshine calculations. A detailed discussion of the resuspension pathway model is contained in Appendix VI of the RSS.

\subsubsection{Ingestion of Radionuclides From Directly Contaminated Crops}

This pathway is considered only for the year following the occurance of an accident and results from deposition of radioactivity on plant surfaces. Ingestion of iodine. $S r$ and $C s$ is considered for milk and ingestion of $S r$ and $C s$ is considered for crops other than milk. Appendix VI of the RSS contains a detailed discussion of how ingestion exposure is related to ground concentrations.

This exposure pathway is excluded if any actions such as land interdiction or land decontamination are taken. Land decontamination assumes removal and destruction of contaminated crops. This pathway is also excluded if doses to a critical individual as a result of the pathway exceed predetermined dose limits. CRAC2 uses dose limits for each of the elements considered for both consumption of milk and consumption of "other" crops ("other" crops are assumed to be crops other than milk). The criteria used for milk are for doses to children and include: (1) 3.3 rem to the bone marrow in the first year for sr: 3.3 rem to the whole body for $\mathrm{Cs}$; and $10 \mathrm{rem}$ to the thyroid for Iodine. The levels used for other crops are for any member of the population and include: 2.0 rem to the bone marrow in the first year for $\mathrm{Sr}$ and 2.0 rem to the whole body for Cs. Based on the 
dose criteria, consumption of milk and/or consumption of "other" crops produced in the area may be prohibited. If radioactivity levels in the area are sufficiently low (i.e.. if doses received by an individual who is assumed to consume nothing but food produced in the area do not exceed the criteria immediately following the accident), the CRAC2 code assumes consumption of milk or "other" crops continues uninterrupted. If the initial activity is too high, the code determines whether or not radionuclide decay and weathering can reduce the contamination to the acceptable level. If these waiting periods do not result in sufficient reduction of potential doses. then the food pathway being considered (i.e.. milk or "other" crops) is assumed to be destroyed. The waiting periods considered are 60 or 30 days for "other" crops. depending on the time in the growing season. and anywhere from 0 to 90 days for milk consumption.

Doses to organ $\ell$ in the time interval $i$ after the accident that result from ingestion of other crops are calculated using the following general equation.

$\operatorname{DSCOM}(4, \ell, i)=\sum_{j=1}^{3} \operatorname{CSING}(i, \ell, j) \cdot K+\sum_{j=1}^{2} \operatorname{sRING}(i, \ell, j) \cdot K$.

where

$$
\begin{aligned}
K= & {[0.85 \cdot \exp (-0.693 \cdot \operatorname{TWAIT} / \operatorname{TEFF}(j))+} \\
& 0.15 \cdot \exp (-0.693 \cdot \operatorname{TWAIT} / \operatorname{HALF}(j))] \cdot \operatorname{CF}(j, 1) \cdot G C(j \cdot k) .
\end{aligned}
$$

and

$$
\operatorname{TEFF}(j)=14 \cdot \operatorname{HALF}(j) /(14+\operatorname{HALF}(j))
$$

The time intervals after the accident are 0-10, 10-20, 20-30.

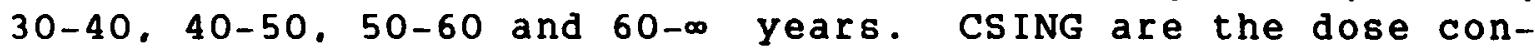
version factors for three $C s$ isotopes (Cs-134. Cs-136 and Cs-137 are treated) and SRING are the dose conversion factors for two $S r$ isotopes ( $S r-89$ and $S r-90$ are treated). The conversion factors have units of rem/Ci ingested. TWAIT is the waiting period and can be $0.0,30.0$, or 60.0 days. GC and HALF are as previously defined and the CF factors relate ground concentration to ingestion by an individual. These CF factors assume that an individual consumes food only from the contaminated area. These factors have units of $\mathrm{Ci}$ ingested/Ci/m².

Doses resulting from milk ingestion to organ $l$ in the time interval $i$ after the accident are calculated using the following general equation. 
$\operatorname{DSCOM}(3, \ell, i)=\sum_{j=1}^{3} \operatorname{CSING}(i, \ell, j) \cdot K+\sum_{j=1}^{2} \operatorname{SRING}(i, \ell, j) \cdot K+$

$$
\sum_{j=1}^{2} \operatorname{RIING}(i, l, j) \cdot K
$$

where

$$
\begin{aligned}
K= & {[0.85 \cdot \exp (-0.693 \cdot \operatorname{TIMER} / \operatorname{TEFF}(j))+} \\
& 0.15 \cdot \exp (-0.693 \cdot \operatorname{TIMER} / \operatorname{HALF}(j))] \operatorname{CF}(j, 2) \cdot \operatorname{GC}(j, K) .
\end{aligned}
$$

and

$$
\operatorname{TEFF}(i)=14 \cdot \operatorname{HALF}(j) /(14+\operatorname{HALF}(j))
$$

The time intervals are the same as those for crop ingestion. RIING is the dose conversion factor for two iodine isotopes (I-131 and I-133 are considered). TIMER is the waiting period and can be from 0.0 to 90 days. The $C F(j, 2)$ factors relate ground concentration to ingestion by an individual who consumes milk produced only in the area. the units are $\mathrm{Ci}$ ingested/Ci/m $\mathrm{m}^{2}$.

\subsubsection{Ingestion of Radionuclides Via Root Uptake}

The model also calculates doses resulting from ingestion of foods contaminated via root uptake. The code calculates these doses for both milk consumption and "other" crop pathways. The radionuclides that are treated include Sr-89, Sr-90, Cs-134 and Cs-137. This exposure pathway is ignored if the area is assumed to be permanently interdicted. Otherwise, exposures are determined from time equal to 0.0 or 1.0 year: when the decontamination factor. DEC. which was determined during the groundshine calculations, is greater than 1.0, and then doses are determined from 1.0 year.

The doses to an organ $\ell$ in time interval $i$ which result from milk ingestion are calculated using the following general equation:

$\operatorname{DSCOM}(5, \ell, i)=\sum_{j=1}^{4} \operatorname{RTING}(i, \ell, j) G C(j, k) \cdot \operatorname{CF}(5, j)$

$$
\cdot \exp (-0.693 \cdot \operatorname{TIDEC} / \operatorname{HALF}(j)) / D E C
$$


The RTING factors for the 4 nuclides relate ingestion exposure to dose and have units of rem/Ci ingested. TIDEC is either 0.0 or 365 days. DEC is the decontamination factor that was determined during the groundshine calculations, and the $C F(5, j)$ factors relate ingestion via milk to ground concentration. The doses from "other" crop ingestion are determined using the following equation:

$$
\begin{aligned}
\operatorname{DSCOM}(6, l, i)= & \sum_{j=1}^{4} \operatorname{RTING}(i, l, j) \cdot \operatorname{GC}(j, k) \cdot \operatorname{CF}(6, j) \\
& \cdot \exp (-0.693 \cdot \operatorname{TIDEC} / \operatorname{HALF}(j)) / \operatorname{DEC}
\end{aligned}
$$

The CF $(6, j)$ factors are used to relate ingestion of "other" crops to ground concentrations of radionuclides for the root uptake pathway. The time intervals considered in the calculation of DSCOM are 0-10, 10-20, 20-30,30-40,40-50, and

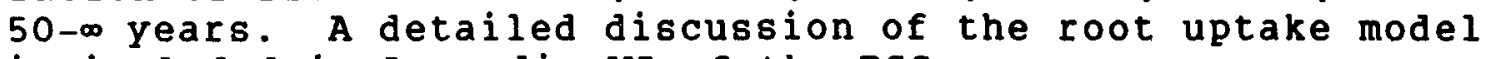
is included in Appendix VI of the RSS.

\section{References for section 3.3}

1. WASH-1400(1975), Reactor Safety Study, Appendix VI: Calculation of Reactor Accident Consequences. NUREG 75/104. US Nuclear Regulatory Commission.

2. Trubey, D. K. and Kaye. S. V. (1973), The EXREM I I I Computer Code for Estimating External Radiation Doses to Populations from Environmental Releases. Oak Ridge National Laboratory. ORNL-TM-4322.

3. Slade. D. H. (Ed)(1968), Meteorology and Atomic Energy. USAEC TID-24190. 
Table 3.3-1 Early Time Periods Used to Evaluate Acute Internal Dose to Organs

\begin{tabular}{|c|c|}
\hline Organ & Time Period \\
\hline Lung & 1 Year \\
\hline Bone Marrow & 30 days* \\
\hline $\begin{array}{l}\text { Endosteal Cells, Stomach Wall. } \\
\text { Small Intestine. Upper Large } \\
\text { Intestine. Lower Large Intestine }\end{array}$ & 7 days \\
\hline Whole Body & 2 days ${ }^{\star \star}$ \\
\hline
\end{tabular}

* Internal bone marrow doses received within the time period of 7 to 30 days after the initial exposure would be only half as effective as that received in the first 7 days.

* The two day internal whole body dose. plus one-third of any internal whole body dose delivered at a rate greater than 20 rads per day. 
Table 2-7. Nuclidea Involved In Chronic Exposure Calculatione

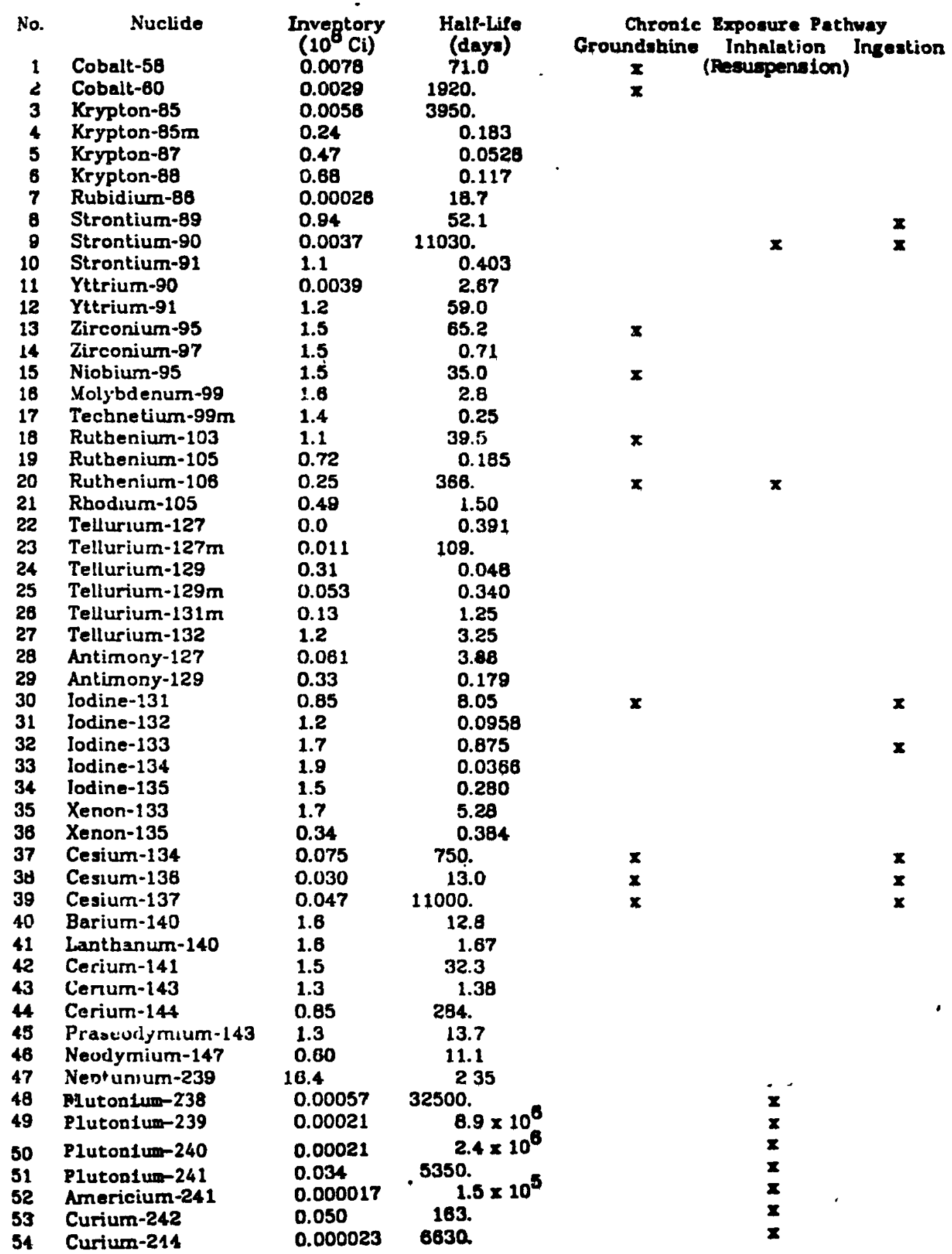

All 54 radionuclides enter the early exposure calculatione, 


\subsection{Emergency Response Model}

The CRAC2 model of emergency response* represents an extensive revision of the corresponding RSS Model. Three types of protective measures are incorporated into the emergency response model of CRAC2. These measures include evacuation. sheltering. and early relocation. These measures are discussed in the following sections. The model allows the user to specify up to six different emergency response scenarios with their respective probability of occurrence. The weighted sum of these scenarios represents the "summary" emergency response. The user can thus specify a range of emergency response alternatives and their summary.

\subsubsection{Evacuation}

Warning times for an impending significant release of radioactive material could vary from essentially none to several hours, and several more hours might pass before the released plume would reach a particular population group, depending on the windspeed following the release. Due to this available time, evacuation is given considerable attention as a public protective measure in most current radiological emergency preparedness programs in the United states. Evacuation is potentially the most effective method of avoiding radiation exposure and can provide essentially total protection if completed prior to arrival of the plume. Two different evacuation models are available as options in the CRAC2 computer code. The first evacuation model is based on a constant "effective" evacuation velocity with no delay time following evacuation notification. This model was used in the Reactor safety study[1]. The second evacuation model is based on a constant evacuation velocity plus a delay time between evacuation notification and initiation. This model was used in the nuclear reactor siting study[2]. Both of these models are discussed in the following sections.

\subsubsection{Reactor Safety Study Evacuation Model}

The Reactor safety study (RSS) evacuation model is based on an "effective" evacuation velocity with no assumed delay time between being notified to evacuate and actually initiating evacuation. For those distance intervals being evacuated (e.g.. from 0 to 25 miles). this evacuation model calculates the distance at which a radioactive plume will overtake persons evacuating from each distance interval using the following formula:

\footnotetext{
*The emergency response model described in this section is implemented in subroutine EVAC and parts of subroutine EARLY.
} 


$$
\text { DIS }=\text { VEL } \cdot(\text { MID + (EVAC }- \text { WARN })) /(\text { VEL-EVAC }) .
$$

where

$$
\begin{aligned}
\text { DIS = } & \text { distance from the site at which the plume } \\
& \text { overtakes evacuating persons from a given } \\
& \text { distance interval (meters). } \\
\text { VEL = } & \text { average plume or wind velocity between the site } \\
& \text { and midpoint of the distance interval being } \\
& \text { evacuated through (meters/second). } \\
\text { MID = } & \text { distance between the site and the midpoint of } \\
& \text { the distance interval where evacuation was } \\
& \text { initiated (meters). } \\
\text { EVAC = } & \text { evacuation velocity (meters/second). } \\
\text { WARN = } & \text { effective warning time (seconds): i.e. the } \\
& \text { time between beginning evacuation and the start } \\
& \text { of the atmospheric release. }
\end{aligned}
$$

This distance is calculated for each distance interval from which evacuation is initiated. If this calculated distance is beyond the maximum evacuation distance considered in the calculation, evacuating persons from the given distance interval from which evacuation was initiated are assumed to receive no radiation exposure. If this distance where evacuees are overtaken by the cloud is within the maximum evacuation distance. the evacuees are assumed to be exposed to the entire duration of the cloud (cloudshine and inhalation) at this distance and to the ground exposure for 4 hours.

\subsubsection{Revised Evacuation Model}

An improved model of public evacuation was developed to provide a more adequate evaluation of evacuation as a radiological emergency response[3]. The revised treatment incorporates a delay time before public movement. followed by evacuation radially away from the reactor at a constant speed. Different shielding factors and breathing rates are used while evacuees await evacuation or are being evacuated. All persons within the designated evacuation area are assumed to move as a group at the same speed after an assumed delay time. No consideration is given to the possibility of a nonparticipating segment of the population. This latter assumption results in upper bound estimates of evacuation effectiveness. given a specific delay time and velocity. The evacuation effectiveness would decrease linearly with an increasing nonparticipating fraction of the population. Evacuating persons are assumed to move to a 
specified distance from the site and are then assumed to avoid further exposure to the radioactive plume.

The revised evacuation model also calculates more realistic exposure durations to airborne and ground-deposited radionuclides than the RSS evacuation model. The RSS emergency evacuation model employs an exposure model for an instantaneous point source such that all released plumes have zero effective lengths. Because of this. evacuating persons overtaken by the plume in the RSS evacuation model are exposed to the entire cloud at the point they are overtaken by the cloud. However. a released plume of radioactive material would have a finite release duration and a length that depends on the wind speed during and following the release. A person overtaken by the front of the plume might still escape before being passed by the entire plume and thus would receive only a fraction of the full plume exposure. It is also possible that an evacuating person may travel under the cloud for a long time and thus receive more exposure than if he had remained stationary during the passage of the plume. The revised evacuation model assigns the plume a finite length which is calculated using the assumed release duration and wind speed during the release. To simplify the treatment, the length of the cloud is assumed to remain constant following the release (i.e.. the front and back of the plume travel at the same speed), and the concentration of radioactive material is assumed to be uniform over the length of the cloud. The radial position of evacuating persons, while stationary and while in transit, is compared to the positions of the front and back of the plume as a function of time to determine a more realistic period of exposure to airborne radionuclides. The revised treatment calculates the time periods during which people are exposed to radionuclides on the ground while they are stationary and while they are evacuating. Because radionuclides would be deposited continually from the plume while passing a given location. a person while under the plume would be exposed to ground contamination which is less concentrated than if the cloud had completely passed. To account for this. the revised model assumes that persons completely passed by the plume are exposed to the total ground contamination calculated to exist after complete passage of the plume. to one-half the calculated ground contamination when anywhere under the cloud, and to no concentration when in front of the plume.

\subsubsection{Population sheltering}

In this model, non-evacuating people living within the maximum sheltering distance are sheltered using the shielding factors defined for the sheltering region. Sheltering, as used by this emergency response model, is defined as the deliberate action by the public to take advantage of the protection against radiation exposure afforded by remaining indoors. away from 
doors and windows, during and after the passage of the radioactive plume. The shielding inherent in normally inhabited structures offers some degree of protection against external penetrating radiation from airborne and surface-deposited radionuclides. Furthermore, the exclusion of a significant amount of airborne radioactive material from the interior of a structure, either by natural effects or by certain ventilation strategies. can reduce the amount of inhaled radionuclides as well. The shielding provided by a structure against external penetrating radiation from airborne or surface-deposited radionucldes is expressed in terms of a shielding factor (SF) which is the ratio of the dose received inside the structure to the dose that would be received outside the structure.

\subsubsection{Population Relocation}

Population relocation is defined in this emergency response model to be early relocation of non-evacuating people following deposition. The model provides two alternatives: relocation at a specified time following the deposition: or relocation at 7 days following the deposition. conditional on a bone marrow dose that exceeds $200 \mathrm{rem}$. Population relocation is a postaccident protective measure designed to limit radiation exposure from radionuclides deposited on the ground and other surfaces. since relocation is a post-accident response, it can be implemented in a more selective manner than an immediate evacuation. In many instances, external exposure to contaminated surfaces would. in a relatively short time. result in a dose much greater than the dose due to the other exposure pathways.

\section{References for section 3.4}

1. WASH-1400(1975), Reactor Safety study, Appendix VI: Calculation of Reactor Accident Consequences. NUREG 75/014. US Nuclear Regulatory Commission.

2. Aldrich, D.C.. et al.(1981). Technical Guidance for siting Criteria Development. NUREG/CR-2239, SAND81-1549, Sandia National Laboratories.

3. Aldrich, D. C.. et al.(1978), A Model of Public Evacuation for Atmospheric Radiological Releases. SAND78-0092, Sandia National Laboratories. 


\subsection{Health Effects Model}

This section discusses how CRAC2 evaluates the health effects that might result from the radioactivity released during a reactor accident. Two general classes of health effects are estimated: early health effects and latent health effects. Early health effects include deaths and injuries that might result from large, acute radiation doses. The CRAC2 code uses the estimates of early dose discussed in section 3.3.1 to evaluate early effects. Latent health effects include latent cancer fatalities. genetic effects and thyroid effects. These effects are determined for the radiation doses resulting from early exposure (see section 3.3.1) and for radiation doses resulting from the chronic exposures discussed in section 3.3.2. The calculation of early effects is discussed in section 3.5.1. The calculation of late effects is discussed in section 3.5.2. The health effects models used in CRAC2 are unchanged from the CRAC health effects models described in the Reactor safety study[1]. Changes have been made, however, to the latent cancer risk factor data. The new CRAC2 data reflects the lifetime risk of latent cancer from radiation exposure.

\subsubsection{Early Health Effects}

Two types of early health effects are considered in CRAC2. early fatalities and early injuries. The models used for early health effects* are described in section 9.2 of Appendix VI of the RSS[1].

\subsubsection{Early Fatalities}

Early fatalities are estimated on the basis of acute radiation exposure to the bone marrow. lungs, and gastro-intestinal (GI) tract. The dose-response relationship for early fatality due to bone marrow irradiation is shown in Figure VI 9-1 of the RSS. The dose-response relationship for early fatality due to lung irradiation is shown in RSS Figure VI 9-3. The doseresponse relationship for early fatality due to gastro-intestinal tract irradiation is shown in RSS Figure 9-4.[1] The dose response relationships are implemented in CRAC2 by interpolation of piece-wise linear approximations to the three curves.

The total probability of early fatality is calculated in CRAC2 from the probability of early fatality due to irradiation

\footnotetext{
*The early health effects model described in this section is implemented in subroutine EARLY and part of the DAMAGE subroutine.
} 
of the bone marrow. lung, and gI tract. If the probability of early fatality associated with these three organs is Pl. P2, and $\mathrm{P} 3$. respectively, the total probability of early fatality. $P$. is calculated as:

$$
\mathrm{P}=\mathrm{P} 1+(1-\mathrm{P} 1) \mathrm{P} 2+(1-\mathrm{P} 1)(1-\mathrm{P} 2) \mathrm{P} 3
$$

which is the probability calculated by assuming that these effects are independent (i.e.. no interactions or synergisms).

\subsubsection{Early Injuries}

Early injuries are defined as those illnesses requiring medical attention or hospital treatment. Early injuries are estimated on the basis of acute radiation exposure of the whole body. lungs. and lower large-intestinal wall. The dose-response models for early injuries are described in Appendix VI, section 9.2 .3 of RSS[1].

The total probability of early injury is calculated in the same manner as for early deaths. However, only persons who did not suffer early death are considered.

\subsubsection{Latent Health Effects}

As mentioned earlier, CRAC2 estimates latent health effects* for both early and chronic radiation exposures. The discussion below is broken down into subsections: latent cancer fatalities. thyroid effects and genetic effects. Each subsection will discuss how effects are estimated for early and chronic exposures in terms of the computer code implementation.

\subsubsection{Latent Cancer Fatalities}

Two models are available for estimating latent cancer fatalities. the linear hypothesis method and the central estimate method. The linear hypothesis assumes a linear relationship between radiation doses and latent cancer fatalities. The central estimate method assumes a stepwise linear relationship between doses and latent cancer fatalities. This relationship is based on the assumption that lower doses and lower dose rates to an individual will result in a reduced risk of cancer per unit dose of radiation. These models are further described below.

\footnotetext{
*The latent health effexts model described in this section is implemented in the DAMAGE subroutine and part of subroutine EARLY.
} 

3.5.2.1.1 Latent Cancer Fatalities Resulting From Early
Exposure

Section 3.3.1 discussed how the EARLY subroutine accumulates early exposures and doses. The EARLY routine also evaluates the latent cancer fatality risk to an individual that results from early exposure. This $r$ isk is determined for each spatial interval on an organ by organ basis. Organs considered for latent effects are not necessarily the same as those considered for acute effects.

If the RSS central estimate model is to be used to evaluate the risk of cancer from doses to an organ. early doses to the organ are evaluated and are compared to threshold values. Central estimate factors may be applied depending on whether or not the early doses exceed the threshold values. The following equations are used to calculate risk to an individual with the central estimate model: $\operatorname{TOTLAT}(i, 1)=\frac{\operatorname{MRCON}(i, i) / \operatorname{AACTOR}(i)}{\operatorname{MrCL}(i, 1)+\operatorname{CLOUD}(i)+\operatorname{GROUND}(i)]} \cdot$

$\operatorname{TOTLAT}(i, k)=\operatorname{AIRIN}(i, k) \cdot \operatorname{MRCON}(i, k) / \operatorname{FACTOR}(i)$, for $1<k \leq 9$.

The first equation calculates the risk of cancer to an individ. ual due to early groundshine and cloudshine doses to organ $i$ and to doses to organ i received during the first year as a result of inhalation from the plume. The second equation calculates risk due to plume inhalation doses to organ $i$ in later time intervals $\mathrm{k}$. These intervals are 1-10, 10-20, 20-30, 30-40. 40-50, 50-60, 60-70 and 70-80 years. The variables are defined as follows: AIRIN is the inhalation dose, CLOUD is the early cloudshine dose. GROUND is the early groundshine dose. MRCON is the risk factor and FACTOR is the central estimate modification factor.

The central estimate factor. FACTOR, is determined for orqan $i$ by using the following series of equations.

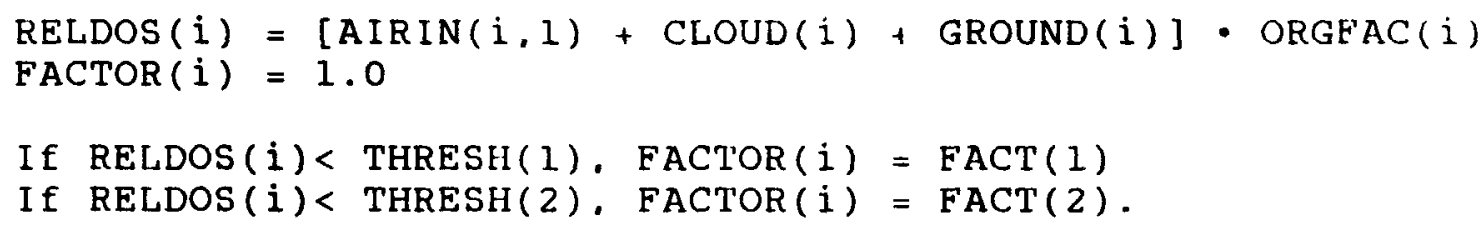

The variable ORGFAC is used to modify the central estimate model for specific organs. The central estimate model may be overridden for a specific organ by imputing a large number for 
ORGFAC. The variables THRESH and FACT are input to the CRAC2 code and are usually defined as follows: THRESH(1) $=300 \mathrm{rem}$. $\operatorname{FACT}(1)=2.5$. THRESH(2) $=30 \mathrm{rem}$, and FACT $(2)=5.0$. The central estimate model is discussed further in Appendix $V I$ of the RSS[1]. For situations where the linear model is elected. the FACTOR values are always set to 1.0 .

Table 5.2-1 shows data used by the CRAC2 code to calculate latent cancer fatalities. The risk factors are updated versions of those reported in the RSS. The RSS factors assumed a latency period during which the risk of cancer was assumed to be zero. followed by a risk period where the individual is assumed to be at a constant risk ( $r$ isk plateau). Depending on the type of cancer and the age of the exposed individual, the latency periods ranged from 0 to 15 years and the risk periods ranged from 10 to 30 years. Based on recommendations in BEIR III[2]. the factors used in CRAC2 were to reflect extension of the risk period to the end of an individual's life for all cancers except leukemia and for all age groups (of exposed individuals) other than those exposed in utero.

The 0 to 1 year conversion factors are used for doses due to external exposure and doses due to chronic inhalation and ingestion. The linear model is always used for the estimation of $r$ isk due to breast cancer. A detailed discussion of the concepts behind the derivation of the risk factors and concepts behind their use is given in Appendix VI of the RSS[1].

For a given weather sequence and spatial interval, the risk to an individual surviving early death is determined as follows.

$$
\text { TOTLE }=\sum_{i=1}^{N}\left(\sum_{k=1}^{9} \operatorname{TOTLAT~}(i, k)\right)
$$

The variable $N$ is the number of organs (i.e.. cancer types) considered and $k$ is the index over the time intervals. For each weather sequence and plume trajectory, the total numbers of latent cancer deaths due to early exposure is determined by summing the products of interval risk and interval populations at risk. The population at $r$ isk in a given interval is determined by the plume widths and wind direction. Only that portion of the exposed interval populations that are not early fatalities is used to calculate cancer fatalities.

\subsection{Latent Cancer Fatalities Resulting From Chronic Exposure}

Section 3.3.2 discussed how CRAC2 calculates chronic radiation doses. The chronic exposure model in CRAC2 
accumulates long-term doses due to groundshine. inhalation of resuspended materials and ingestion of contaminated crops and milk. The number of latent cancer fatalities that might result from these different types of doses are calculated using the 0 to 1 year risk factors shown in Table 3.5-1. This calculation is performed for each weather sequence at each spatial interval. The effects that are calculated for each spatial interval are modified if the central estimate model is employed. The central estimate factor that is employed is the one determined for the estimation of latent cancer fatalities due to the early exposure pathways. As noted earlier. those factors are determined at each spatial interval for each weather sequence.

The number of cancer fatalities occurring in time period $k$ due to chronic doses to organ $i$ is calculated using the following equation.

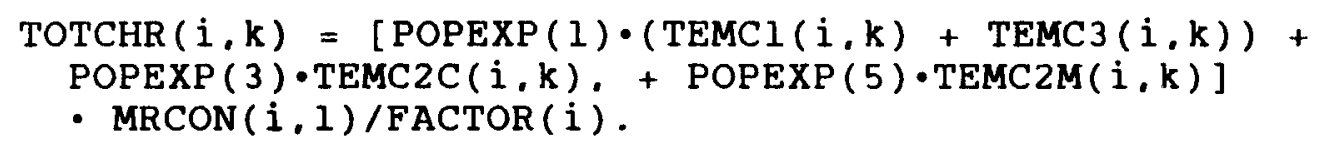

TEMC1. TEMC2C. TEMC2M, and TEMC3 are the individual dose commit ments in rem for chronic inhalation of resuspended material. crop ingestion, milk ingestion, and groundshine, respectively. For a given interval, POPEXP(1) is dependent upon the width of the plume and the site population distribution. This population is the number of people within the interval that are within the contaminated area. POPEXP(3) and POPEXP(5) are the populations that are exposed via ingestion of crops and of milk, respectively. These populations are based on the agricultural production in the areas covered by the plume and are not necessarily the populations residing in these areas. MRCON is the risk factor and FACTOR is the central estimate modification factor.

The numbers of latent cancer fatalities resulting from chronic exposure in the different intervals are summed for each weather sequence. The total number of latent cancer fatalities for a weather sequence is determined by adding the fatalities for early exposure and the fatalities for chronic exposure.

\subsubsection{Thyroid Effects}

The CRAC2 code calculates the total number of thyroid nodules that would result from early exposure doses and from chronic doses received in the interval from 0 to 1 year. Early exposure doses to the thyroid are treated somewhat differently from the rest of the organs. In addition to early groundshine and cloudshine, three categories of inhalation dose are computed: I-131. all other iodines, and all other nuclides. When calculating the number of thyroid nodules. CRAC2 treats the $I-131$ 
dose from early inhalation as being only 10 percent as effective as external irradiation. This 10 percent portion of the I-13I thyroid dose is summed with the thyroid doses resulting from other radionuclides to obtain an effective early exposure dose for calculating effects. If the total thyroid dose is less than $1500 \mathrm{rem}$. the total number of nodules is equal to the effective early exposure dose times the dose conversion factor. If the total thyroid dose is between 1500 and 5000 rem. the total number of nodules predicted with the dose conversion factor is cut in half. If the dose is above $5000 \mathrm{rem}$, there will be no nodules (thyroid ablation). This model is further discussed in section 9.3.5 of Appendix VI of the RSS[1].

CRAC does not apply the 0.1 effectiveness factor to the I- 131 inhalation dose from the chronic exposure pathways. For chronic doses, total number of nodules is equal to the product of total man-rem and the risk factor. CRAC2 assumes that 334 nodules will result per $10^{6}$ man-rem. A more complete discussion of the concepts behind the above model is found in Appendix VI of the RSS[1].

The following equations describe how CRAC estimates the individual risk of having a thyroid nodule for early exposure.

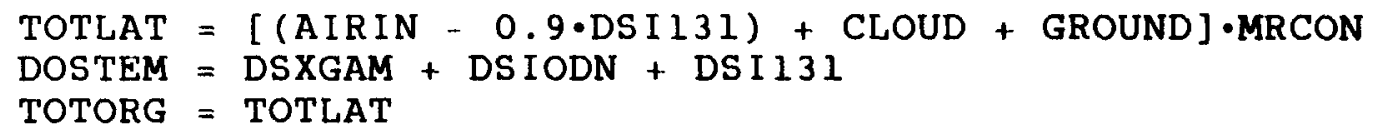

In this case, TOTLAT is the individual risk of having a thyroid nodule. MRCON is the risk factor ( 334 nodules/106 man-rem). AIRIN is the total inhalation dose received within 1 year. DSII31 is the one year dose from I-131, and CLOUD and GROUND are the early external doses due to cloudshine and groundshine. respectively. The one year inhalation doses from other iodines. DSIODN, and from other gamma emitters, DSXGAM, are summed with DSII3I to determine the magnitude of the total thyroid dose. The calculated risk per individual is applied to the surviving exposed population that lies within the interval to determine the number of nodules.

The CRAC2 code also calculates the number of nodules that can result from chronic doses received in the interval from 0 to 1 year. In this case, inhalation doses due to I-131 are not treated with reduced effectiveness. This calculation is done 
in the same manner as the calculation of latent cancer fatalities for chronic exposure. The following general equation is employed.

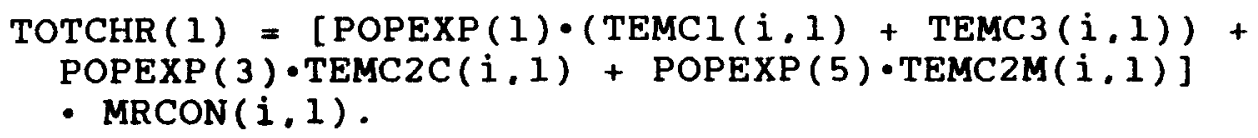

The general definitions of the variables in this equation are the same as those for the latent cancer fatality calculations. However, organ $i$ represents the thyroid and the risk factor. $\operatorname{MRCON}(i, 1)$. is for total thyroid nodules (334 nodules/106 man-rem).

\subsubsection{Genetic Effects}

CRAC2 can also calculate the incidence of genetic effects provided that the proper data are input. This is done by replacing the latent cancer fatality data with that given in Table 3.5-2. The data given in this table are taken from the input file for the original CRAC code. The effects treated by these factors are single-gene disorders. multifactorial disorders. chromosomal disorders and spontaneous abortions. A discussion of these effects is found in Appendix VI of the RSS[1].

When calculating these effects, the CRAC2 code would use the same equations and procedures as used for latent cancer fatalities. However, ingestion doses from chronic exposure are ignored. In this case. the MRCON values are those shown in Table 3.5-2 and the risk modification factor. FACTOR, is equal to 1.0. Testes is the organ considered.

\section{References for section 3.5}

1. WASH-1400 (1975). Reactor Safety Study, Appendix VI: Calculation of Reactor Accident Consequences. NUREG 75/014. U. S. Nuclear Regulatory Commission.

2. BEIR (Committee on the Biological Effects of Ionizing Radiation), (1980). The Effects on Populations of Exposures to Low Levels of Ionizing Radiation. National Academy of sciences, Washington. DC. 
TABLE $3.5-1$

Data Used by CRAC2 to Calculate Latent Cancer Fatalities

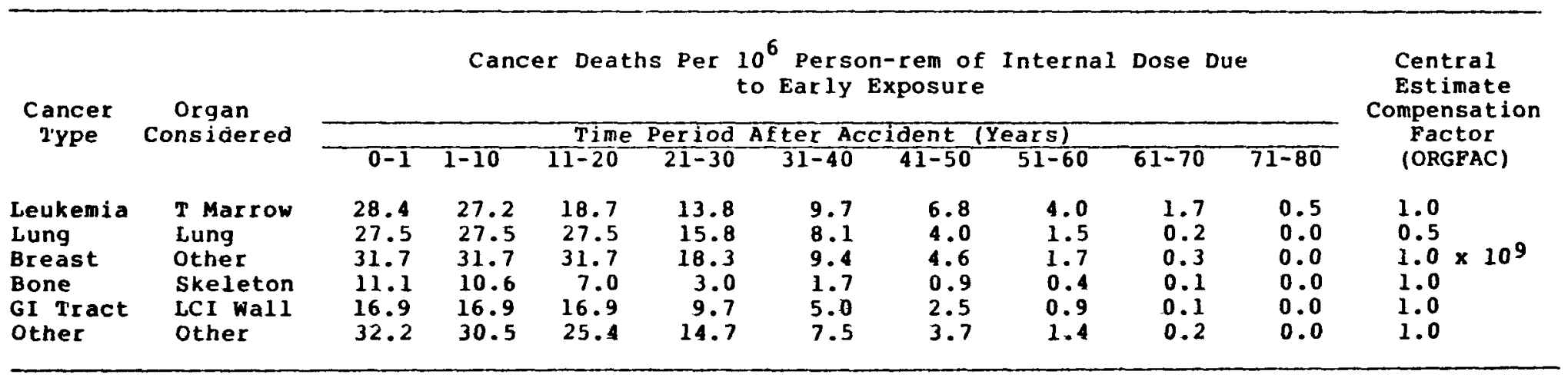


TABLE 3.5-2

Data Used to Calculate the Incidence of Genetic Disorders and Spontaneous Abortions

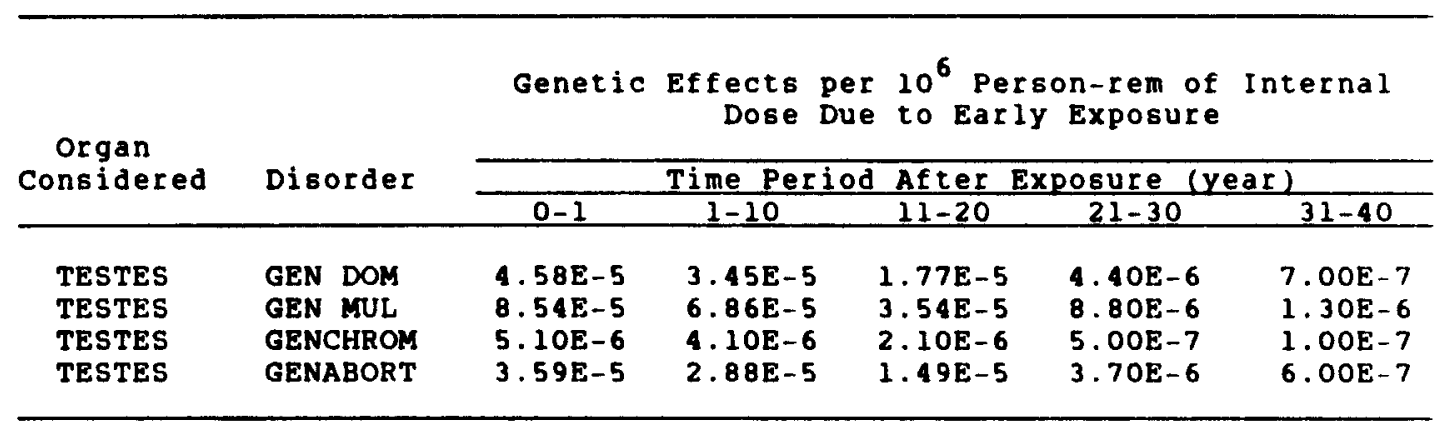




\subsection{Economic Effects Model}

The economic consequence model in CRAC2 estimates the direct costs of measures taken to mitigate the public health effects of a reactor accident. The model estimates the cost of evacuation. milk and crop disposal, decontamination, and land use prohibition (interdiction) which may be required after an accident. Supporting economic data associated with these costs are supplied as input data. The model is identical in structure to the original CRAC economic model described in the Reactor safety study (RSS) [1].

The term evacuation is used in this discussion to refer to the immediate movement of individuals out of an area at the time of the accident. This is distinguished from relocation. which is the movement of the population from an area for a protracted period of time. Land interdiction refers to the prohibition of inhabitation or use of land in an area for a period of time ranging from months to years.

\subsubsection{Costs of Acute Mitigative Actions-Evacuation}

The estimation of evacuation costs in the CRAC2 economic model is based on the number of individuals evacuated. The population evacuated is based on the keyhole-shaped evacuation area described in the RSS or on a circular evacuation area. The costs of evacuation are computed by multiplying the total number of individuals evacuated by an average cost per evacuee. The cost per evacuee includes evacuation supervision costs, transportation costs, and food and lodging costs. The average evacuee cost is estimated in the RSs based on an assumed 7-day evacuation period. This cost has been updated using cost component inflators to reflect the 1980 cost in 1980 dollars.

\subsubsection{Costs of Long Term Mitigative Actions}

The remaining costs estimated in the economic model are dependent upon the consequence mitigation measures implemented in each specified spatial interval. Long-term protective measures are determined by the radioactivity deposited in each region after the accident. User-defined criteria which specify the acceptable time-integrated dose levels are used to determine the measures to be implemented in each spatial interval. Each spatial interval is assigned to one of six mutually exclusive long-term protective action categories:

1) Land area interdiction, for some period of time, followed by land and property decontamination 
2) Immediate decontamination of area followed by inhabitation

3) Milk and crop disposal

4) Crop disposal

5) Milk disposal

6) No long-term actions

One long-term action alternative is assigned to each spatial interval based on the deposited radioactivity and acceptable integrated dose criteria specified. Costs are estimated ror each spatial interval based on the selected long-term action alternatives. Total costs are estimated through summation over all spatial intervals including acute evacuation and long-term protective action costs.

\subsubsection{Land Area Interdiction Followed by Land and Property} Decontamination

Land area interdiction is considered as a likely consequence mitigation measure if the ground activity deposited at the time of the accident is unacceptable based on the time-integrated dose criterion, and decontamination efforts would not be successful in lowering the ground deposited radioactivity to an acceptable level for immediate inhabitation. In this case. it is assumed that the use of the land for normal activities would be forbidden for at least some time to allow for decay and weathering of deposited radionuclides. It is also assumed that the annual production of crops and milk would be disposed of if the accident occurs during the growing season and ground activity level exceeds an acceptable level for immediate inhabitation. The economic model includes the cost of disposal of farm products. the decontamination costs, time value losses on land and property, and relocation costs in the total cost estimates for land interdiction.

The cost of farm products disposal is estimated by using the equation:

$$
\operatorname{COST}(1)=\text { APFARM・SEASF . }
$$

where

$$
\begin{aligned}
\text { APFARM = } & \text { state average annual production of rarm products } \\
& \text { multiplied by the number of acres of farmland } \\
& \text { contaminated. }
\end{aligned}
$$




$$
\begin{aligned}
& \text { SEASF }=0 \text { if accident occurs outside the state growing } \\
& \text { season } \\
&=1 \text { if accident occurs within state growing } \\
& \text { season. }
\end{aligned}
$$

The decontamination costs are estimated using cost estimates derived in the RSS which have been updated to 1980 dollars [2]:

$$
\operatorname{COST}(2)=(E F F A R M \cdot D C F L D)+(E F P O P \cdot D C R B P)
$$

where

$$
\begin{aligned}
& \text { EFFARM = contaminated farmland (acres) in spatial } \\
& \text { interval } \\
& \text { DCFLD = updated maximum decontamination cost ( } \$ / \text { acre) } \\
& \text { for farmland (maximum decontamination factor of } \\
& 20 \text { assumed) } \\
& \text { EFPOP = population living in contaminated area before } \\
& \text { accident } \\
& \text { DCRBP = updated decontamination cost (\$/person) for } \\
& \text { residential, business, and public areas } \\
& \text { (maximum decontamination factor of } 20 \text { assumed) }
\end{aligned}
$$

The cost estimates used for decontamination are based on an assumed decontamination factor between 2 and 20 . The costs of decontamination are never discounted.

Another cost component included in the estimate of land interdiction cost is the value lost due to the restriction of the use of the land for the time until decontamination. decay, and weathering of radionuclides cause the radioactivity deposited to reach an acceptable level. The cost of the land and property value lost is estimated using the equation:

$$
\operatorname{cosT}(3)=\left[1-\left[e^{-r T_{i n t}}\left((1-a)+a \bar{e}^{-d T_{i n t}}\right)\right] v_{0}\right.
$$

where

$$
\begin{aligned}
V_{0}= & \text { total market value of contaminated land plus } \\
& \text { improvements before accident occurrence }
\end{aligned}
$$




$$
\begin{aligned}
a= & \text { fraction of the total market value } v_{0} \text { which is } \\
& \text { in improvements } \\
\mathrm{d}= & \text { assumed depreciation rate of improvements during } \\
& \text { interdiction period }(\mathrm{d}=.20) \\
\mathrm{r}= & \text { interest rate plus property tax rate }(\mathrm{r}=.12) \\
\mathrm{T}_{\text {int }=} & \text { interdiction period. measured from accident } \\
& \text { occurcence until restoration of land use }
\end{aligned}
$$

It is assumed that a cost would be incurred because all indivi. duals would be relocated from the interdicted land area.

Cost estimates used for relocation are those developed in the RSS, indexed to 1980 dollars. These costs are based on a 90-day relocation period involving individual unemployment and wage losses, individual and business transportation and moving costs. and lost business profits. Total relocation costs are estimated using the equation:

$$
\cos T(4)=E F P O P \cdot C R E L O C
$$

where

$$
\begin{aligned}
\text { EFPOP }= & \text { population previously living in the } \\
& \text { contaminated area } \\
\text { CRELOC }= & \text { relocation cost ( } \$ / \text { person) estimated in the } \\
& \text { RSS. updated by indexing to } 1980 .
\end{aligned}
$$

Finally, the economic model compares the cost of decontamination and interdiction with the alternative option of land interdiction for a longer period with only weathering and decay of deposited radionuclides to an acceptable level. The following two options are compared:

Option I : Land interdiction followed by decontamination

$$
\text { Total cost }=\cos T(1)+\cos T(2)+\operatorname{cosT}(3)+\cos T(4)
$$

Option II: Land interdiction for longer period allowing for radioactivity decay and weathering

$$
\begin{aligned}
\text { Total cost }= & \operatorname{cosT}(1)+\operatorname{cosT}(4)+\left[1-\left[e^{-r T} d / w(1-a)+\right.\right. \\
& \left.\left.a e^{-d T / w}\right]\right] v_{0}
\end{aligned}
$$


where

$$
\begin{aligned}
\mathrm{T}_{\mathrm{d} / \mathrm{w}}= & \text { time required for deposited radioactivity to } \\
& \text { weather and decay to an acceptable level without } \\
& \text { decontamination }
\end{aligned}
$$

The option with the least total cost for a particular spatial interval is chosen and used in estimating economic costs of land interdiction. The total cost of land interdiction in a spatial interval is the sum of the estimates of farm product disposal costs, decontamination costs (if employed), land and property time value costs, and relocation costs.

\subsubsection{Immediate Decontamination and Inhabitation}

Immediate decontamination and inhabitation of an area is assumed to occur if the deposited level is initially unacceptable and decontamination can reduce the contamination to an acceptable level immediately. In this case. it is assumed that the annual production of farm products would be disposed of if the accident occured in the growing season. The cost of farm product disposal is estimated using equation ( 1 ).

Decontamination costs are estimated using the following equation:

$\operatorname{COST}(2)=($ EFFARM·DCFLD $)+($ EFPOP.DCRBP $)$ DECO

where

$$
\begin{aligned}
\mathrm{DECO}= & 1 \text { if the decontamination factor necessary to attain } \\
& \text { an acceptable activity level is greater than } 2 . \\
\text { DECO = } & 0.4 \text { if the decontamination factor necessary to } \\
& \text { attain an acceptable activity level is less than } 2 .
\end{aligned}
$$

The cost factor DECO is intended to account for the reduced cost of decontamination estimated in the RSS for decontamination by a factor of less than 2 .

The total cost of immediate decontamination and inhabitation of a land area is the sum of the farm product disposal costs and the decontamination costs. No relocation costs or time value losses are incurred in this case.

\subsubsection{Farm Product Disposal}

If deposited radioactivity levels are acceptable for immediate land inhabitation but doses from ingestion of milk and 
crops in the growing season at the time of the accident are unacceptable. then it is assumed that the annual crop production is disposed of, and milk is disposed of until the milk ingestion dose becomes acceptable. This cost is estimated using the equation:

$$
\operatorname{COST}(1)=(\text { APCROP }+ \text { APMILK・TDISP }) \text { SEASF }
$$

where

$$
\begin{aligned}
\text { APCROP }= & \text { state average annual farm production of crops } \\
& \text { (\$/acre) multiplied by the number of acres of } \\
& \text { farmland contaminated } \\
\text { APMILK = } & \text { state average annual milk production ( } \$ / \text { acre) } \\
& \text { multiplied by the number of acres of farmland } \\
& \text { contaminated } \\
\text { TDISP = } & \text { time period during which dose for ingestion of } \\
& \text { milk would be unacceptable }
\end{aligned}
$$

The variable SEASF is defined as in equation (1). If the accident occurs outside the growing season it is assumed that cows were fed with stored feed, and no losses are incurred. No decontamination, relocation, or time value losses are incurred in this case.

\subsubsection{Crop Disposal}

If deposited radioactivity levels are acceptable for immediate inhabitation, but doses from ingestion of crops in growing season at the time of the accident are unacceptable, then it is assumed that the annual crop production is disposed of. In this case, the doses from ingestion of milk are unacceptable. The cost of crop disposal is estimated using the equation:

$$
\operatorname{COST}(1)=\operatorname{APCROP} \cdot \mathrm{SEASF}
$$

where the variables have been previously defined. No decontamination relocation, milk disposal, or time value losses are incurred in this case.

\subsubsection{Milk Disposal only}

If deposited radioactivity levels are acceptable for immediate inhabitation and crop ingestion. but doses from ingestion of milk are unacceptable, then it is assumed that milk would be disposed of if the accident occurs during the growing season. The cost of milk disposal is estimated using the equation: 


$$
\operatorname{COST}(1)=(\text { APMILK TDISP }) \text { SEASF }
$$

where the variables have been previously defined. No decontamination, location, crop disposal, or time value losses are incurred in this case.

3.6.2.6. No Long-Term Actions

In areas where the deposited radioactivity does not cause any of the acceptable dose limits to be exceeded, it is assumed that post-accident protective actions and economic conditions have no significant associated direct costs.

$$
\text { References for section } 3.6
$$

1. WASH-1400 (1975). Reactor Safety Study. Appendix VI: Calculation of Reactor Accident Consequences. NUREg 75/014. US Nuclear Regulatory Commission.

2. Aldrich, D.C.. et al. (1982). Technical Guidance for siting Criteria Development, NUREG/CR-2239 (SAND81-1549), Sandia National Laboratories. 


\section{APPENDIX A}

The CRAC2 Computer Code

The purpose of this appendix is to describe the CRAC2 computer code that implements the models described in section 3 of this report. Every attempt has been made to ensure an accurate rendition of these models with the goal of providing a consistent and reliable computer code.

The description of the computer code consists of two parts. an overview of the concept of operation of the code, and a description of the design and flow of the code elements. 


\section{A. 1 Concept of Operation}

CRAC2 incorporates a progression of mathematical and statistical models which represent the radioactive material immediately after release from containment; the movement of the material as it disperses into the area around the power plant: the deposition of the material by wet and dry deposition processes and the effects of the material on man and his environment. The code approaches the calculation by dividing the area around a power plant into radial annuli which are called spatial intervals. The spatial interval is basic to these computational processes. Figure A-l depicts a power plant and this spatial interval concept. The code allows for a maximum of 34 such spatial intervals.* The program computes the average concentration and total coverage of the radioactive cloud for each spatial interval. All other mathematical and statistical models are processed in terms of these spatial intervals.

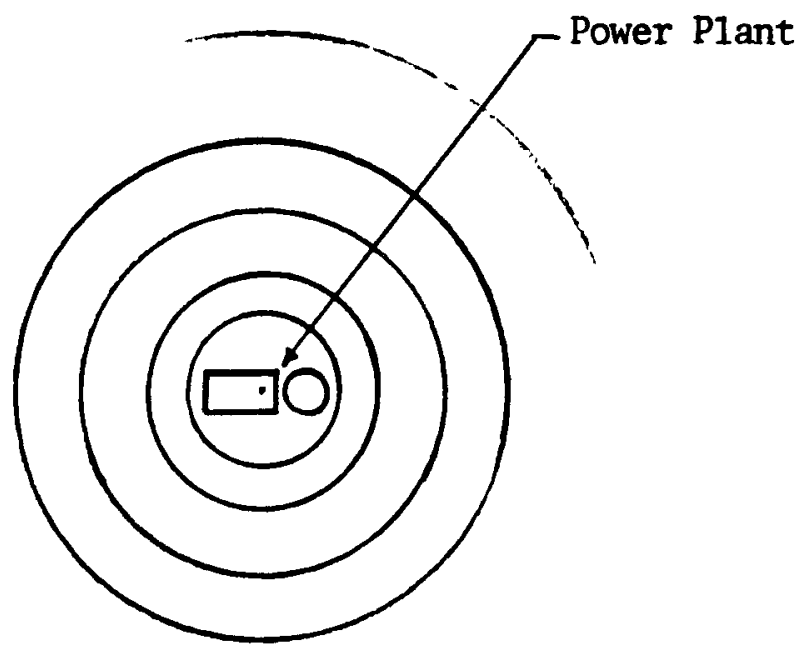

Figure A-1 spatial Interval Representation

* See Table IV 4-1 in Appendix VI of the Reactor Safety study. 
The operation of the CRAC2 code functions can be outlined in terms of six logical steps. These steps divide the computational model into code segments that represent distinct models or recurrent processes.

Problem definition is the first step in the process. This step occurs once for each problem case specified by the user.

1. Problem Definition. The computational process is initialized by defining the problem to be solved. The INPUT control routine and fifteen input subroutines process the input data subgroups that define the user's problem.

The next two steps must be repeated for each new weather sequence, but are independent of the accident sequence.

2. Meteorological Conditions.* The second step in the computational process assigns the specific meteorological conditions to each spatial interval. This is done either in input subroutine SITE when user supplied meteorology is to be incorporated, or in subroutine SPADAT when time dependent meteorological data have been specified. A thermal stability. wind speed. and precipitation rate are assigned to each interval. This allows for changes to occur in the dispersion pattern of the cloud of radioactive material. A meteorological file containing representative hourly weather data can be automatically sampled and hourly meteorological data assigned if requested by the user.

3. Dispersion.** The next step incorporates the meteorological data into the Pasquill-Gifford model of Gaussian dispersion. This step involves the calculation of standard deviations of the horizontal and vertical dispersion values, oy and $\sigma_{z}$. and isotope dependent decay constants at the midpoint of each spatial interval. This computational step is performed in subroutine DISP.

For each weather sequence, the final three steps must be repeated for each accident sequence: up to this point the steps have been independent of the accident sequence.

\footnotetext{
* See Chapter 5 of Appendix VI of the Reactor safety study

* See Chapter 4 of Appendix VI of the Reactor safety study
} 
4. Activity.* This step computes the air and ground concentrations of each radionuclide for each spatial interval. The accident parameters are utilized to compute plume rise and any initial isotope decay. The cloud is also depleted by wet and dry deposition mechanisms. All calculations are done at the midpoint of the spatial intervals. Air and ground concentrations are uniform over the entire interval area which has been contaminated by the cloud of radioactive material. Subroutine ACTIVE is responsible for this step.

5. Damage.** The damage step assesses the impact of the contamination upon man and his environment. The damage model determines how far the people in a specific spatial interval will evacuate before the cloud overtakes them. The dose to the organs of these people is computed from the immediate or early exposure. Fatalities and injuries are the resultant consequences. The latent dose is computed for the survivors and an assessment is made of the latent cancer fatalities. Chronic exposure doses are computed and a determination is made of the protective action for the contaminated land. Costs associated with this action are then computed. The population groups are factored into the results so that consequences represent each population sector. Subroutines DAMAGE. EARLY. CHRONX and PRPDAM are responsible for the damage calculations.

6. Results. Each set of consequences is stored and final statistics are computed for all desired results.

subroutines STORE and STOROPT provide these functions.

* See Chapter 4 of Appendix VI of the Reactor Safety study.

* * See Chapters $8,9.11$, and 12 of Appendix VI of the Reactor safety study. 


\section{A. 2 Program Flow}

The CRAC2 code consists of a main program, a control routine for input processing and fifteen input subroutines. eleven meteorology and dispersion subroutines and nine damage and results subroutines. A description of each subroutine is given below. The code itself contains extensive comments to supply the user with sufficient information to read and work with the program.

\section{A.2.1 Program MAIN, the CRAC2 Code Controller}

The MAIN routine controls the execution of the CRAC2 code based upon the directions of the user. This routine calls subroutine INPUT to process the set of data that defines the problem to be solved. MAIN determines how many sets of results and/or meteorological sequences have been specified for this study and calls subroutine SITE to load the desired meteorolo-gical and site data. The control routine calls a sequence of routines for the computation of each meteorological sequence.

1. Subroutine SPADAT - Calculates the meteorological conditions for each spatial interval.

2. Subroutine DISP - Calculates the dispersion parameters at the midpoint of each spatial interval.

3. Subroutine ACTIVE - Calculates the air and ground concentration of the radionuclides at the midpoint of each spatial interval.

4. Subroutine DAMAGE - Calculates the health effects and property damage for all spatial intervals and population sectors.

When all of the meteorological sequences for a specific problem have been processed. the entry point FSUM in subroutine STORE or FSUMOPT in subroutine STOROPT is called to compute the final summaries. MAIN controls the entire sequence of computations until all problems defined by the user have been completed. The flow of the CRAC2 control program is given in Figure $\mathrm{A}-2$.

\section{A.2.2 Subroutine INPUT, the Input Controller}

Subroutine INPUT controls the input processing for CRAC2. The routine reads and prints the title card and processes the subgroup header card. For each subgroup header card read, it then calls the appropriate subroutine to process the corresponding subgroup of data*. Table A-l gives a brief description

* See Chapter 2 of the CRAC2 Computer Code User's Guide. NUREG/CR - 2326 . 
of the fifteen input subroutines that can be called by INPUT. - INPUT also controls the files which maintain and reinitialize the reference data after each problem case. When a group of data is modified, the reference data being changed is stored on file NAT. With the completion of a problem case, the reference data on file NAT is reinitialized and another problem case can be performed. Subroutine INPUT functions in one of three modes: read in the reference case (IREST $=-1$ ): store the reference data and read in the modified data (IREST = 0): or restore the reference case data (IREST $=1$ ). INPUT also terminates the execution of the program when all of the problem cases for this execution of CRAC2 are complete. All of the input subroutines shown in Table A-l follow essentially the following processing scheme. When the reference case is to be input, IREST $=-1$, and the routines read in the appropriate data. When a data subgroup is to be modified. IREST $=0$. and the routines write out whatever data is found in the data arrays to the NAT file and then read in the modification data over the existing arrays. When the reference case is being reinitialized. IREST $=1$, and the routines read whatever data as been written to the NAT file.

\section{A.2.3 Subroutine SITE, the Accident Site Data Processor}

Subroutine SITE is responsible for reading the site characteristic weather data from the meteorological data file and the population and topographical data from the site data file. These data sources are read only as they are requested by the user in the input data from the input subgroups. If the user has requested the importance sampling technique (ISTART $=5$ in the SITE subgroup data) to select the meteorological trials. subroutine SITE calls the BINMET subroutine which sorts the meteorological data into the 29 weather categories provided by the CRAC2 mode1.

\section{A.2.4 Subroutine BINMET, the Meteorological Data Sorter}

Subroutine BINMET sorts the full year of meteorological data representing the site into 29 weather categories (also called bins) selected for the CRAC2 model. Each weather sequence is examined to determine (1) the first occurrence of rain within 30 miles of the site. (2) the first occurrence of a wind speed slow down within 30 miles of the site. or (3) the stability and wind speed at the start of the sequence. The first of these conditions that is satisfied by the sequence determines the weather category to which it is assigned. Tables are constructed of the weather category frequencies and the bin assignment of each weather sequence. This information is used by the importance sampling algorithm. A summary of the meteorological data. including two tables of the meteorological bin statistics derived from the data, are printed by BINMET. 
Subroutine vare $\begin{gathered}\text { Input data } \\ \text { subgroup }\end{gathered}$

1. SPAT SPATIAL
2. SIT

3. Econom

4. POPU

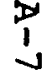

5. TOPO

6. ISOTOP

7. Leakag

e. DIsOPT

SPATIAL

STE

ECONOMIC

POPULATION

TOPOGRAPHY

ISOTOPE

LEAKAGE specifies the radil of ennular

spatial intervals acound the accident ite.

Specifies the site identification

information. the perameters that

def ine the meteorological sampling

meteorological data. and the number

of meteorological trials to be

sanpled. The sources of the

population and topographical data for the site are also defined.

Specifies cost data for computation of economic effects.

Specifies population option switch and the population sectors to be processed. Optionally. the

population for each spatial interval ectors around the site arat ined.

Spectifies the state code and land troction data for each spatiol the site.

Specifies the inventory of isotopes and associated parameters.

specifies the release identification. the associated release parameters. and the fraction of the total core inventory which is released for each isotope leakage group.

DISPERsion specifies the reactor building

dimensions and the secial wake and rain depletion options.
Input Data

Subroutine Nase Subgroup

9. eVacu evacuate

10. ACUTE ACUTE

11. LATE

LATENT

12. CHRON

CHRONIC

13. SCALE

SCALE

14. RESIN RESULTS

15. OPT

OPTIONS
Specifies the emergency protective action parameters.

Specifies the acute effects due to early exposure to the radioactive cloud that are to be studied and the supporting dose-mortality and injury data for each organ.

Specifies the latent effects due to early and chronic exposure that are to be studied and the supporting man-rem conversion factors and the choice of latent

Specifies the data used in radiation doses fron sure and the protective action measures chronic exposure.

Specifies the conseguence magnitude scaling values for tabulating the complementary cunulative distributions of the final results.

Specifies the final results for which mean. variance and complementary cumulative distributions

Specifies the print options for detalled output for each aeceorological trial and the ances control the laten and chronic calculat ons alization. 


\section{A.2.5 subroutine RANBIN, the Latin Hypercube Initializer}

The RANBIN subroutine selects the initial weather sequence sampled from each of the 29 weather categories. The selection is made using the Latin hypercube selection criteria. Latin hypercube sampling is used to assure random samples selected from evenly spaced sets within each bin.

\section{A.2.6 Subroutine SPADAT, the Routine to set spatial Meteorology}

Subroutine sPADAT takes the start time (month, day, and hour) of a given weather sequence and prepares the stability. wind speed and precipitation data based upon the initial and subsequent hourly meteorological conditions. The routine determines the season for this start time. It then stores the proper directional probabilities and the stable and unstable mixing heights for each season. SPADAT assigns a sequence of hourly meteorological data to the spatial intervals by calculating hourly travel distances based upon wind speed. The stability. wind speed and precipitation indicator are assigned to all of those intervals which are covered by the cloud for this hour. If the wind speed for this hour is not sufficient to fully traverse an interval. SPADAT determines the number of hours required for the wind speeds to cover the interval and averages the stability. wind speed and time of precipitation for all of the hours. It then assigns these averaged values to the interval. Months, days, and hours are incremented when necessary by subroutine INCTIM.

A.2.7 Subroutine TIMES, the Generator of stratified Random Times

This subroutine calculates (24) -N stratified random start times. The stratification scheme selects $\mathbf{N}$ random day times and $\mathrm{N}$ random night times from each month.

A.2.8 Subroutine RANDU, the Random Number Generator

RANDU chooses a random number between 0 and 1 for use in computing random start times.

\section{A.2.9 Subroutine TIMES2, the Generator of Random Times}

This subroutine chooses a random start time for the year by selecting a random month, a random day, and a random hour.

\section{A.2.10 Subroutine EXTRCT, the Meteorological Data Extractor}

This subroutine extracts the rain, stability and windspeed data for one hour from the meteorological data supplied for the start code. ISTART $=4$. It then computes the distance traveled by the cloud for the hour. 


\section{A.2.11 Subroutine INCTIM. the Time Incrementing Routine}

This routine increments the hour, day and month counters whenever required.

\section{A.2.12 Subroutine DISP, the CRAC2 Dispersion Model}

The purpose of subroutine DISP is to compute the atmospheric dispersion of the released cloud of radioactive material using the Pasquil1-Gifford parameterization of the Gaussian transport model. The routine is leakage independent and therefore is exercised only once for each set of meteorological conditions processed. DISP is called by the control program one time for each meteorological sequence. Figure $A-3$ shows the flow of the routine.

When it is desired to completely deposit the remnant of the radioactive cloud in the last interval, the option LIRAIN $=34$ is used. The last spatial interval is then enlarged to cover an area out to 2000 miles and rain is forced to fall at the rate of $.5 \mathrm{~mm} / \mathrm{hr}$ over the entire interval.

Each spatial interval has a stability, wind speed and rain rate assigned to it in subroutine SPADAT. The stability and wind speed determine the meteorological forces that will act to disperse the cloud. The lateral diffusion is determined by computing a oy spread based upon the Martin and Tikvart power law relations of the Pasquill-gifford curves. The vertical dispersion calculation of $\sigma_{z}$ is based on a treatment by Turner.

An option is available to reset the values of oy and $\sigma_{z}$ if the building wake is to dominate the plume. Once the values of $\sigma_{y}$ and $\sigma_{z}$ at the midpoint of an interval are known, an initial value of $1 / x$ is calculated. Since the cloud timing to the interval midpoint is available, the exponential isotopic decay constant in the interval for each radionuclide is also computed.

\section{A.2.13 Subroutine ACTIVE, the CRAC2 Activity Mode1}

The principal functions of the subroutine ACTIVE are to compute the air concentration ( $\mathrm{Ci}-\mathrm{sec} / \mathrm{m}^{3}$ ) and ground concentration ( $\mathrm{Ci} / \mathrm{m}^{2}$ ) for each radionuclide at every spatial interval. The control program calls ACTIVE one time for each meteorological sequence. Subroutine ACTIVE incorporates the accident dependent leakage parameters into the calculation for each of the leakage categories defined in the data input.

Figure $A-4$ shows the flow of the routine.

Heat released at the time of the accident lifts the plume of radioactive material off the ground. This plume rise term 
is incorporated into the model by computing a new centerline release height. Brigg's plume rise formulations are incorporated for this purpose. In addition. ACTIVE computes the initial radionuclide decay including daughter buildup from core shutdown to release to the environment.

The calculations performed by ACTIVE are done for each spatial interval. The centerline height, $h$, is computed at the midpoint of each spatial interval as a function of the release height and the plume rise. The exponential term in the Gaussian expression for ground level concentration. EXPON, is computed. as well as the effective cloud height. EFHGHT.

The value of $x$ over $Q$. CHIQ, is computed as the quotient of EXPON and the concentration value generated in the subroutine DISP, modified to reflect the expansion factor associated with the release duration.

The computation from CHIQ of the air and ground concentrations of each radionuclide in the interval considers the amount of radioactive material released into the atmosphere in the accident. the decay and daughter buildup of the radionuclides. and the depletion resulting from dry and wet deposition. These air and ground concentrations are computed at the midpoint of the spatial interval and apply uniformly to the entire area which was covered by the radioactive cloud in the spatial interval.

\section{A.2.14 Health Effects and Property Damage Routines}

The DAMAGE subroutine controls and performs the health effects and property damage calculations. The DAMAGE routine is called by the main program one time for each meteorological sequence. Figure $A-5$ shows the flow within the DAMAGE subroutine.

DAMAGE first assigns the dollar costs of evacuation for each of three evacuation cost models. In the first model no evacuation takes place. In the second model, all people in a circular area surrounding the site are evacuated. The radius of the circle corresponds to the maximum evacuation distance. In the third model, a circular area around the site is evacuated together with an arc that is centered on the prevailing wind direction that extends beyond the circular area. The user must specify the radius of the circle and of the arc as well as the arc width. Costs are calculated based on the number of people evacuated by the respective model.

The accident leakage categories are each processed in the order in which they are defined by the leakage subgroup data. The applicable evacuation cost for the leakage category is assigned as determined by the parameters of the leakage 
category that is being processed. When the warning time is 0 . the first model is used. When the duration of release is greater than EVCosT(4), the second cost model is used: for all other situations the keyhole evacuation cost model is utilized.

The spatial intervals are used as the basis for the health effects and property damage calculation. Subroutines DISP and ACTIVE have previously computed the air concentrations ( $\mathrm{Ci}-\mathrm{sec} / \mathrm{m}^{3}$ ) and ground concentrations $\left(\mathrm{Ci} / \mathrm{m}^{2}\right.$ ) of radioactive material. the area covered by the material, and the cloud duration at each interval. To evaluate the consequences of an accident, DAMAGE processes each spatial interval through (1) an evacuation model: (2) an acute. latent and chronic dose and dose effects model: and (3) a property damage model. A complete set of consequences is computed for each spatial distance from the reactor.

Two options are provided to treat the evacuation of people to mitigate the early exposure of individuals to the radioactive materials released by the reactor accident. The first evacuation option was utilized in the Reactor safety study and is based on a constant effective evacuation velocity. For those intervals being evacuated, e.g.. those intervals within the maximum evacuation distance, the routine calculates the distance required for the cloud to catch the people. The second evacuation option is also based on a constant evacuation velocity. option two. however, incorporates a delay time before public movement, followed by evacuation radially away from the reactor at constant speed. In addition to the maximum evacuation distance, both the assumed delay time and evacuation speed are required as input to the model. Different shielding factors and breathing rates are used while persons are stationary (before evacuation) or in transit (during evacuation). All persons within the designated evacuation area move as a group with the same delay time and evacuation speed.

Both evacuation options provide for sheltering outside of the designated evacuation area. No emergency action occurs outside the sheltering radius. However, shielding factors and breathing rates are defined separately for people who are in the sheltering and no emergency action areas. The sheltering radius must be specified with the input date for the evacuation model.

For both of the evacuation options. the people within the non-evacuating intervals are assumed to remain at their respective locations for either 24 hours. 24 (EXPD) hours or for seven days. In the seven day relocation option, if the seven day dose approaches lethal levels. i.e.. a dose to the bone marrow exceeding $200 \mathrm{rem}$. it is assumed that immediate detection will be made and the people will be relocated after a 24-hour exposure. In the 24 hour or 24 (EXPD) hour relocation options. the people will always be relocated after the specified 24 hour or 24 (EXPD) hour exposure. An exponential interpolation between 
the 8 hour dose and the 7-day dose is performed to calculate this 24 hour or 24 (EXPD) hour dose.

The cloud gamma ray shine factor calculations are performed in routines SHINCF and POL2. This cloud gamma ray shine factor is calculated by a two variable interpolation of the cloud centerline height and vertical standard deviation, $\sigma_{z}$.

The dose and health effects from the early exposure to the cloud are computed next. Early exposure is defined as the exposure from the passing radioactive cloud and from the immediate ground contamination. The passing cloud exposure manifests itself as the dose from the inhalation of the radioactive material and from the cloud gamma ray shine. The immediate ground exposure is based upon an integrated exposure time either during the emergency evacuation or during the relocation phase of the damage calculation.

Subroutine EARLY is utilized to compute these early doses. Figure A-6 shows the flow within this routine. In addition. this subroutine calculates both the acute and latent effects from this early dose. Calculations are performed on the basis of individual spatial intervals.

Subroutine EARLY first calculates the acute effects from the early exposure. For every organ considered. the routine accumulates the dose from each radionuclide. The three exposure paths, cloud shine, inhalation, and ground exposure, are calculated separately and are summed together for the total dose to the organ. Figure A-7 shows a graphic presentation of the modeling of dose with distance from the accident. The dose is represented by a uniform trapezoidal volume for each spatial interval.

Acute dose effects are calculated utilizing a three segment linear interpolation of the dose response curves. Acute effects are the fatalities and injuries which manifest themselves in less than one year after exposure. The calculation of acute effects is performed on an individual organ basis so that the specific cause of the early effects can be determined. The routine generates a probability of acute effect which in turn is accumulated over all organs for this spatial interval. The accumulation process assumes that those people not fatally injured by a previous organ are available to be fatally injured by the next organ. Therefore, a person can only be a fatality one time, by one organ. Synergistic effects between organs are ignored.

The EARLY subroutine also evaluates the latent (long-term) effects from the early exposure when the LAT2 entry point is called. The calculation is again performed on an organ by organ basis. Organs subject to latent effects are not necessarily 
the same as those subject to acute effects. The cloud gamma ray shine and early external ground exposures are accumulated for all isotopes. These exposure modes are one-time (essentially instantaneous) doses. The inhalation dose, on the other hand. is accumulated over the life of the individual. The code calculates the inhalation dose for 10 time periods $(0-1,1-10$, $11-20,21-30,31-40,41-50,51-60,61-70,71-80$ and $>80$ years). A dose conversion factor for each time period up to 50 years is stored and utilized in the effects calculation. For those time periods greater than 50 years, the $41-50$ year dose conversion factor is used. If the central estimate of latent dose effects is to be modeled, the integrated dose within 1 year is modified for the specific organ and then compared against a threshold value. A central estimate factor is then applied if the dose is sufficiently low. The total cases per person for each time period and the summation of cases per person over all time periods is saved for each organ. The total dose effect is computed for all of the organs except for whole body.

The thyroid organ is treated somewhat differently from the rest of the organs. Three categories of dose are computed. the Iodine-131 inhalation dose, the dose from all other iodines, and the dose from all other nuclides. The total thyroid dose is the sum of the three categories. If the total thyroid dose is less than $1500 \mathrm{rem}$. the total number of thyroid nodules is equal to the effective early exposure dose times the dose conversion factor. If the total thyroid dose is between 1500 and $5000 \mathrm{rem}$. the total number of nodules predicted using the dose conversion factor is reduced by 50\%. If the total thyroid dose is above 5000 rem, there will be no nodules: thyroid ablation occurs instead.

The chronic exposure calculation is done in subroutine CHRONX. The calculation is performed on an individual spatial mesh, utilizing the non-evacuating ground concentrations. subroutine CHRONX determines the appropriate measures to be taken for the given amount of ground contamination. The action measures include milk or crop disposal, land decontamination and/or total land interdiction. CHRONX also calculates the dose received from any low level radiation that people are exposed to for long periods of time. Chronic doses are calculated from ground gamma ray shine and from inhalation due to the resuspension and ingestion of radionuclides. CHRONX calculates the chronic doses utilizing the total dose commitment model. Figure A-8 shows the flow for the CHRONX routine. The routine works with groups of isotopes which have been determined to be critical for a given exposure mode. It first calculates the ratio of actual activity to allowable activity for the interdiction criterion. If this ratio is greater than one, the land must be either decontaminated or interdicted. The routine next determines if, after a 30 year period, the radiation levels have decayed sufficiently to allow people to return. If the people can return, the total time for the land interdiction is 
determined by interpolating between the 30 year and 2 year dose levels. When the ratio of actual activity to allowable activity is greater than 20 and the total time for interdiction is greater than 30 years. the land will then be permanently interdicted. Permanent interdiction means the government must take over ownership of the land and ensure that the area is guarded. The factor of 20 represents the maximum decontamination that could be realistically accomplished. If the ratio is less than a factor of 20. the land will be decontaminated and the people will be allowed to return after the land is made habitable. If the ratio after 30 years is greater than 20 but the total time for radiation levels to get to the acceptable levels is less than 30 years, an interdiction time with decontamination is computed.

For those intervals where total interdiction is not required, the CHRONX routine calculates the expected doses the people will receive from each of the applicable pathways. The first pathway is the external irradiation dose commitment. This is calculated for each of the time periods associated with the latent effects. The inhalation dose commitment from resuspension is also calculated for each time period. Crops, dairy products and milk will automatically be destroyed if the ratio of actual to allowable dose commitment is greater than one. If the dose via ingestion of crops and/or milk is deemed to be greater than acceptable. the crops and/or milk will be destroyed. Thus, CHRONX determines the action required for a given amount of activity. This action can either (1) destroy only the milk: (2) destroy only the crops: (3) destroy both the milk and crops: (4) decontaminate the land, and/or interdict it for a relatively short period of time: (5) interdict the land for a minimum 30 years or (6) do nothing. If the crops, dairy products and milk do not have to be destroyed. the dose from the ingestion of these will be calculated. The final computation determines the dose commitment via the plant root uptake and ingestion.

The model assumes that doses, health effects, and measures of property damage are uniform over the entire contaminated area within the spatial interval. This contaminated area within the spatial interval is defined by the spatial radii and three sigma lateral spread of the cloud times the expansion factor (see Figure $A-7$ ).

The population and total dollar values are factored into the calculation for each $221 / 2^{\circ}$ sector. The ratio of the cloud coverage to the sector angle is computed and a determination of the number and fraction of sectors involved is obtained. The cloud path is then rotated through all of the sectors (16) for this spatial interval. This calculation enables the utilization of the dose, health effects and property damage measure for the incorporation of population densities and dollar values 
for all directions about the reactor with an appropriate probability of wind direction factored into the final result.

The economic costs associated with property damage are the final consequences calculated by the DAMAGE subroutine. PRPDAM is called once for each spatial interval to calculate the economic damage caused by the accident. Figure A-9 shows the flow of PRPDAM. For the spatial interval and sector. PRPDAM identifies the state code. population affected, farm land affected. the annual production (per acre) of farm products for the state, annual production (per acre) of dairy products for the state, and the annual production (per acre) of non-dairy products for the state.

The costs for the disposal of milk depend on the annual milk production and the total period of milk disposal. Crop disposal costs are based on disposal of the annual crop production. The milk and crop disposal costs are assumed to be zero for accidents not occurring within the growing season. The costs for decontamination of land areas are based on disposal of the annual milk and crop production. plus land and property decontamination costs for residential, business, and public areas and farm areas. Decontamination costs are computed by multiplying the farm decontamination costs (per acre) by total farm acreage and adding the residential, business, and public property decontamination costs (per person) multiplied by the total population involved. A maximum decontamination factor of 20 is used in determining if interdiction of land areas is required. The costs of land interdiction include depreciation of improvements (e.g.. buildings, tangible wealth) during the interdiction period with the assumption that some portion of the initial value of land and buildings may be recovered at the termination of the interdiction period. Present value discounting is used to calculate the portion of the value of the area which may be recovered after the interdiction period is completed. The depreciation of improvements accounts for the loss of value due to lack of maintenance during the interdiction period. Improvements account for $70 \%$ of the initial value of all residential, business, and public land areas, and 258 of the initial value of all farm areas. Permanent population relocation costs are also added in those areas requiring land interdiction. These costs are computed by multiplying the number of persons affected by the relocation cost per person. In those areas in which decontamination by the maximum factor (20) cannot immediately reduce individual exposures to acceptable levels, a calculation is performed in PRPDAM to determine if decontamination with interdiction is less costly than interdiction for a longer period of time with no decontamination efforts. The least costly option is selected for computing total accident costs. 
A set of ten costs are generated and returned to DAMAGE for the evaluation of economic consequences: 1) evacuation cost:

2) cost of disposal of milk and crops: 3 ) decontamination costs: 4) land interdiction cost with decontamination: 5) relocation cost with decontamination: 6) land interdiction costs without decontamination: 7) relocation costs without decontamination: 8) direct costs (agriculture and evacuation): 9) total costs with decontamination: 10 ) total costs without decontamination. PRPDAM also determines the total number of people exposed through crop and milk ingestion. These values are calculated based upon crop and milk production and the consumption in dollars per person. Finally, the health effects for each organ and time period are computed, and the demographic data for the site are factored into the results.

\section{A.2.15 Subroutine STORE, the Routine to Process standard Results}

STORE serves two functions in the CRAC2 code. The first function is to save the standard results for each of the meteorological sequences. This is accomplished by a call to STORE from subroutine DAMAGE. The second function of STORE is to prepare the final printout of the standard results. This is accomplished by a call to entry point FSUM from the MAIN program. The subroutine computes the mean and variance over the set of weather sequences for each of the named results. In addition. FSUM saves the maximum value for each named result together with the identification of the meteorological sequence which produced it. FSUM also computes and prints the frequency distribution of the final results by grouping them against the magnitude bins constructed by the SCALE subroutine.

\section{A.2.16 Subroutine STOROPT, the Routine to Process special Results}

Subroutine STOROPT serves the same functions as STORE for all of the special result options provided by CRAC2. The functions of entry point FSUMOPT in STOROPT correspond to the functions of the entry point FSUM in STORE for these same special result options. 


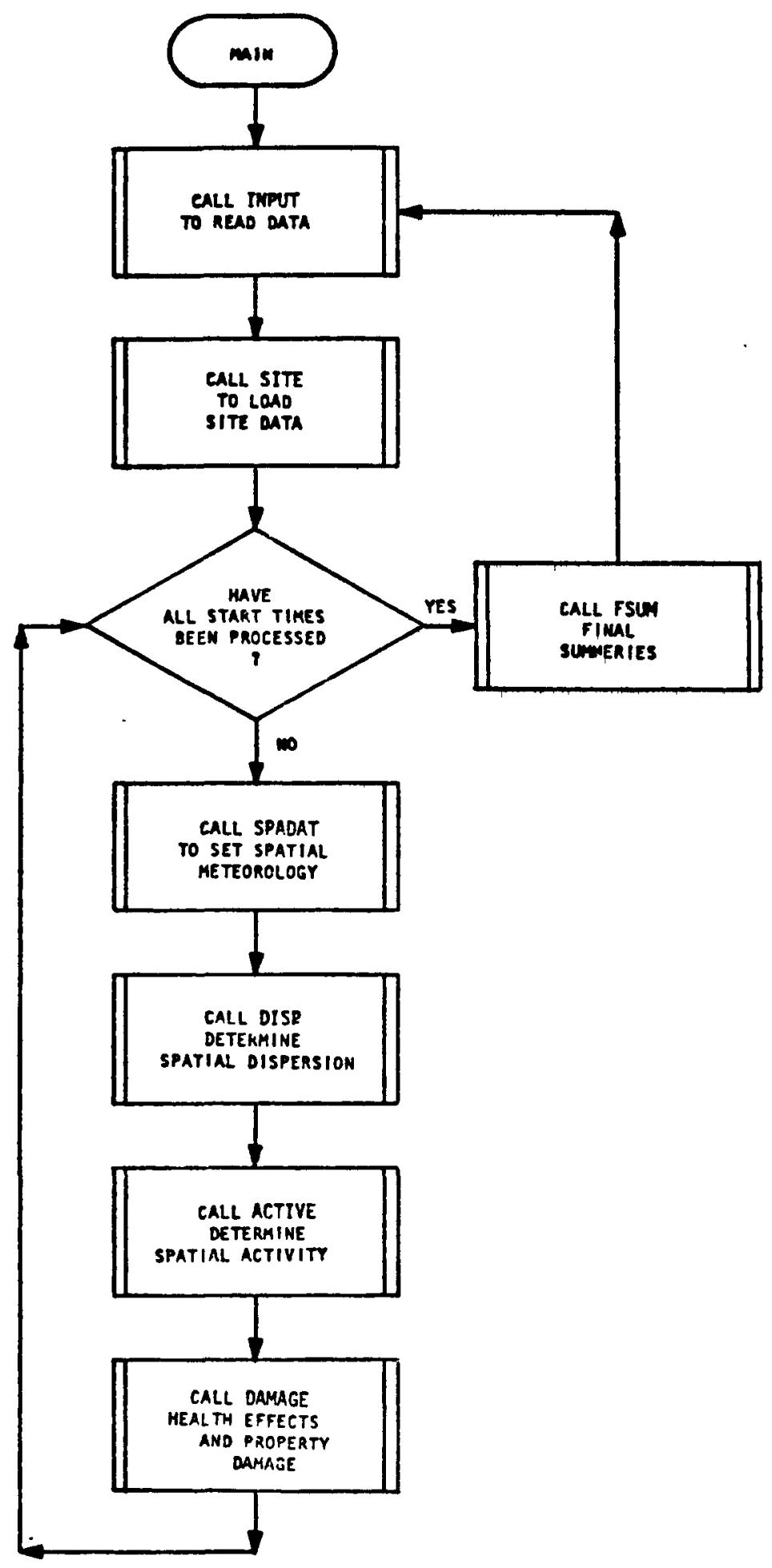

Figure A-2. Flow of MAIN, the CRAC2 Control program

A. 17 


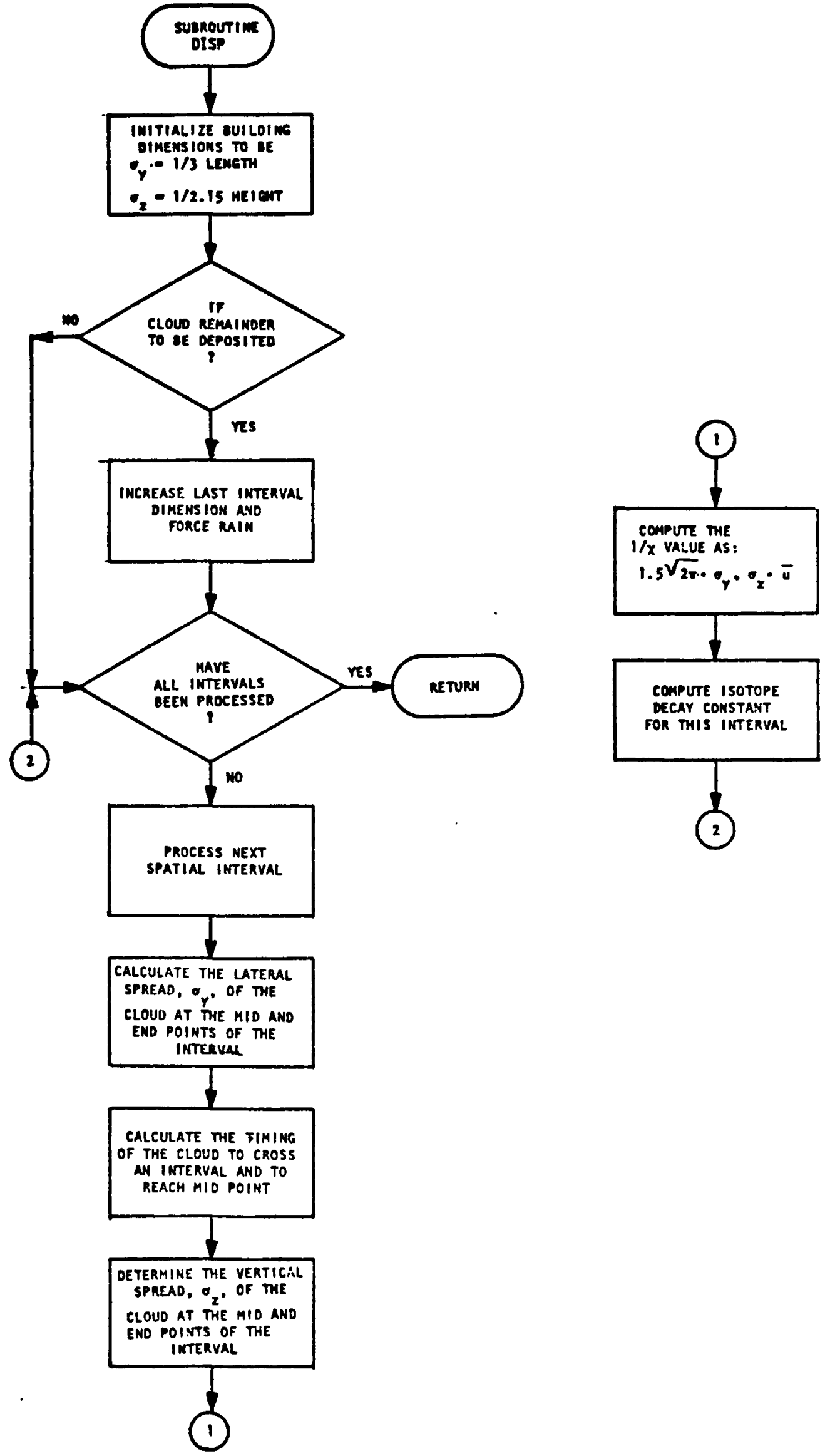

Figure A-3. Flow of DISP, the Dispersion Model 


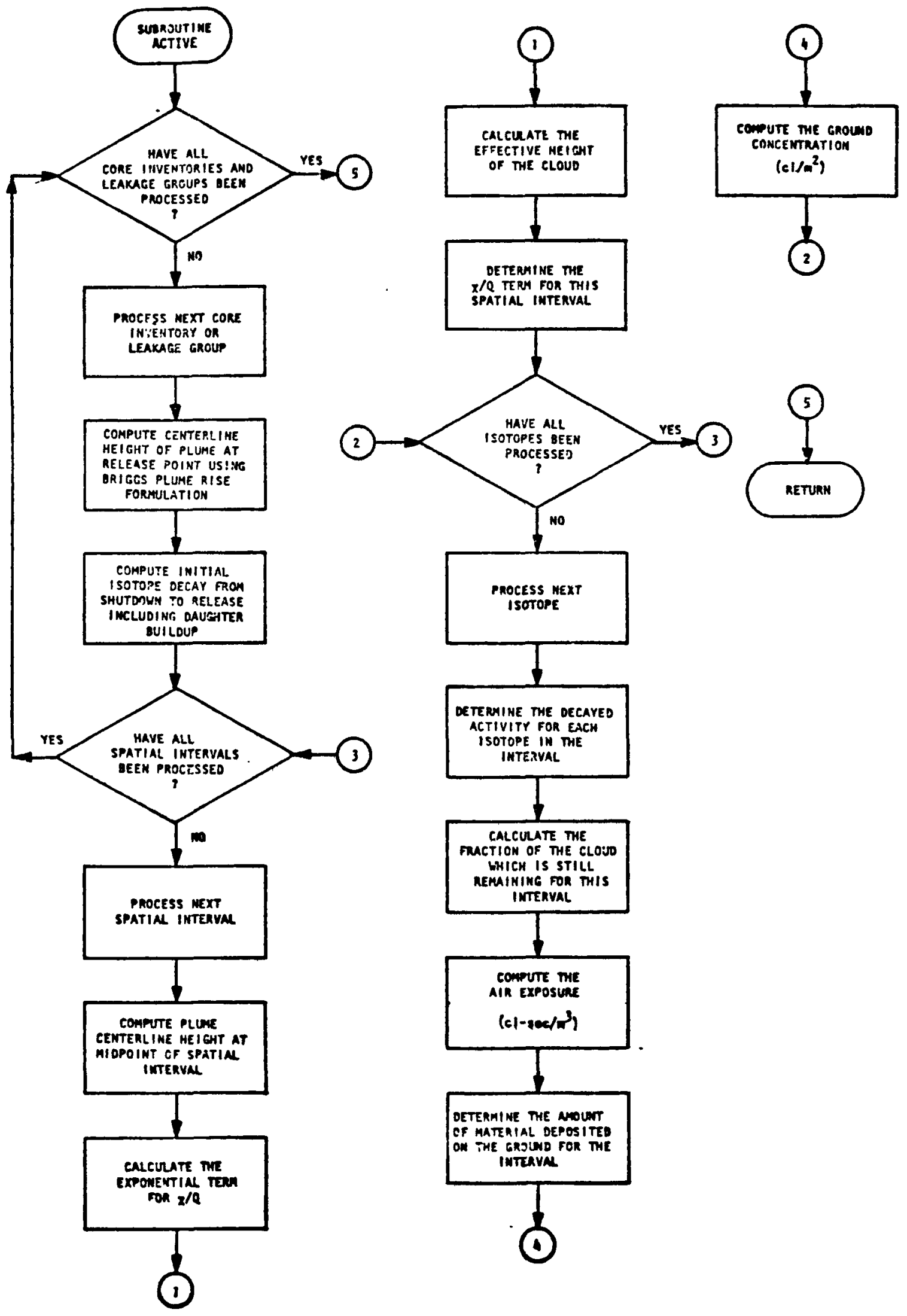

Figure A-4. Flow of ACTIVE, the Activity Model 

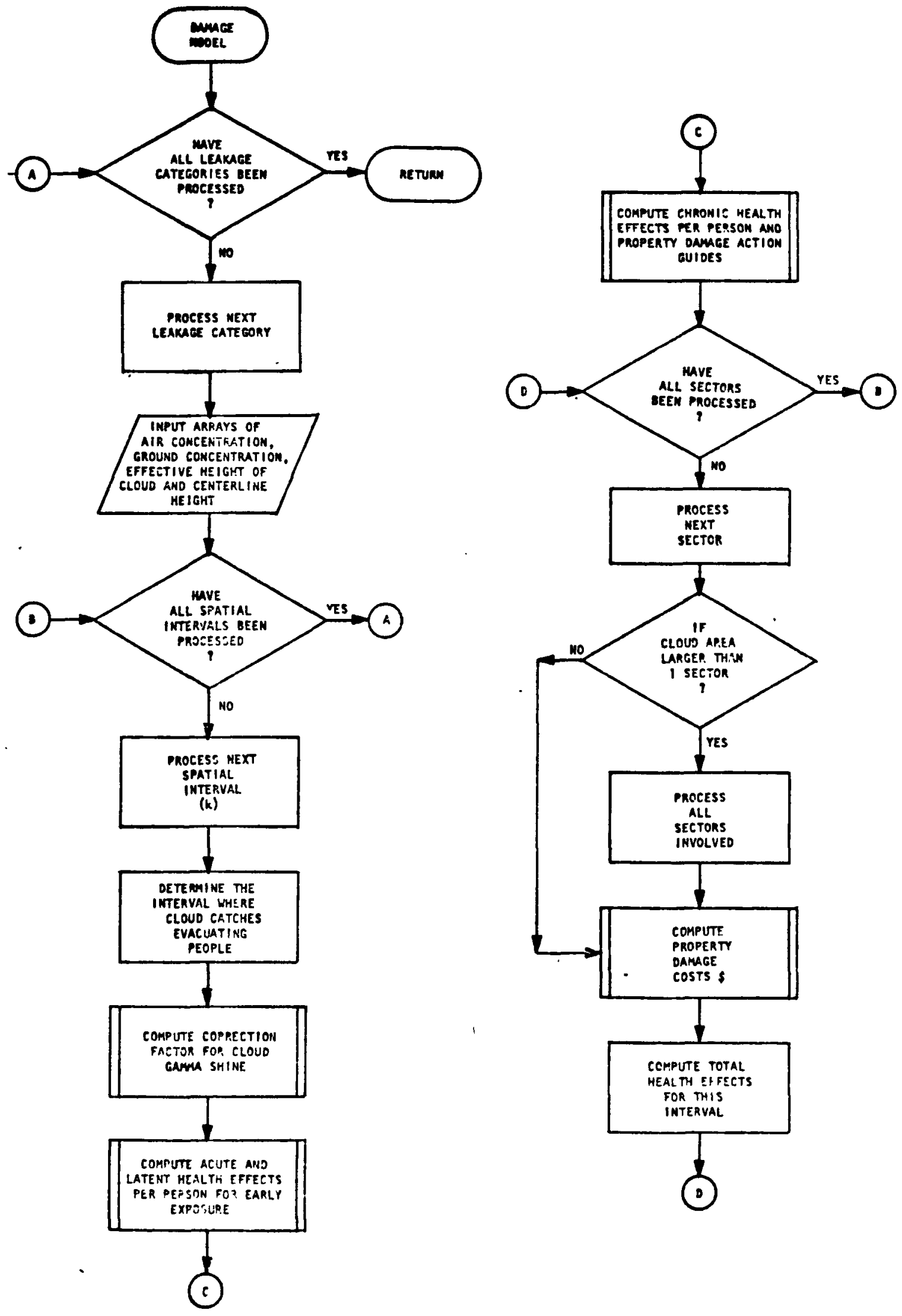

Figure A-5. Flow of DAMAGE, the Damage Model 


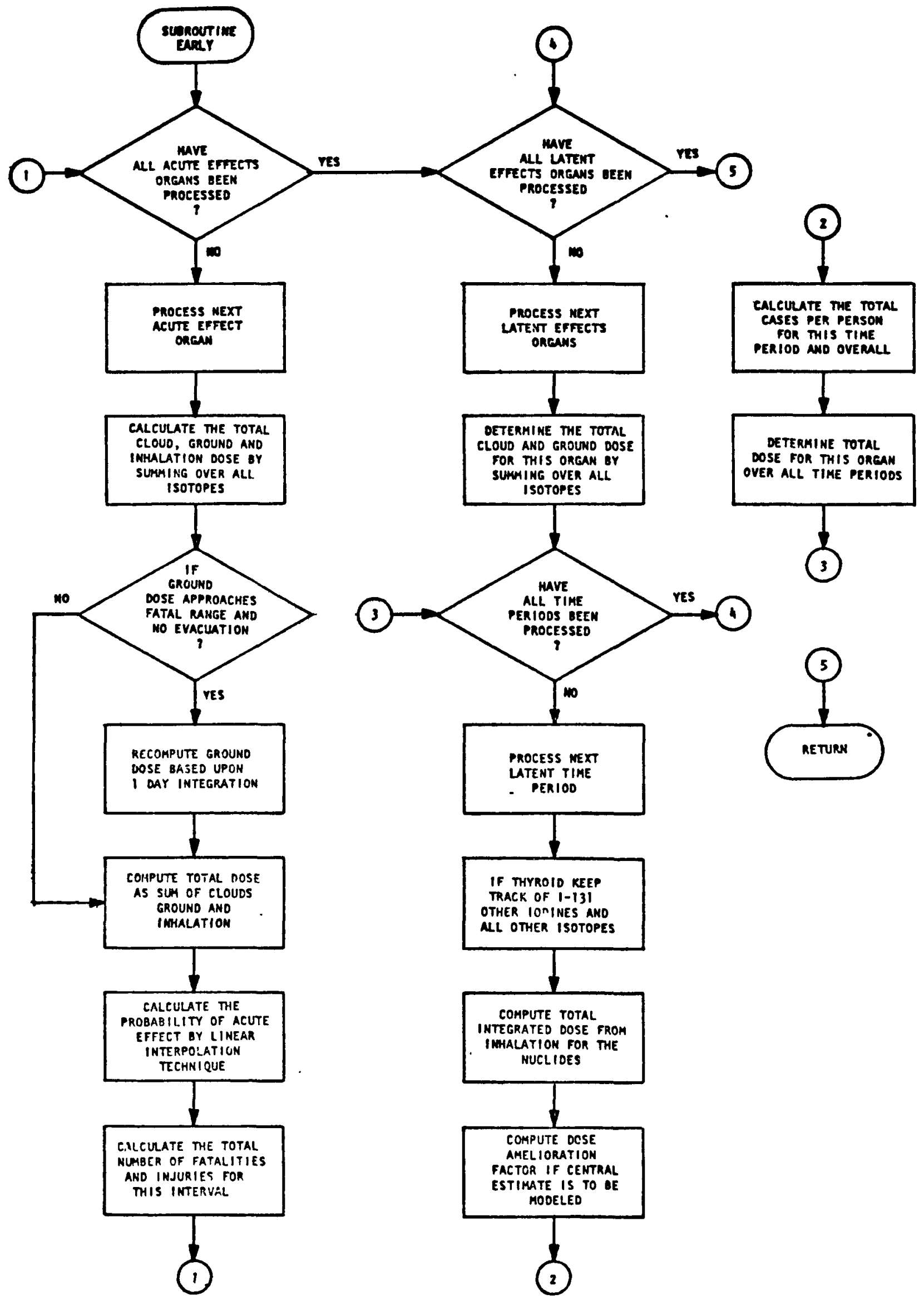

Figure A-6. Flow of EARLY, the Early Effects Model 


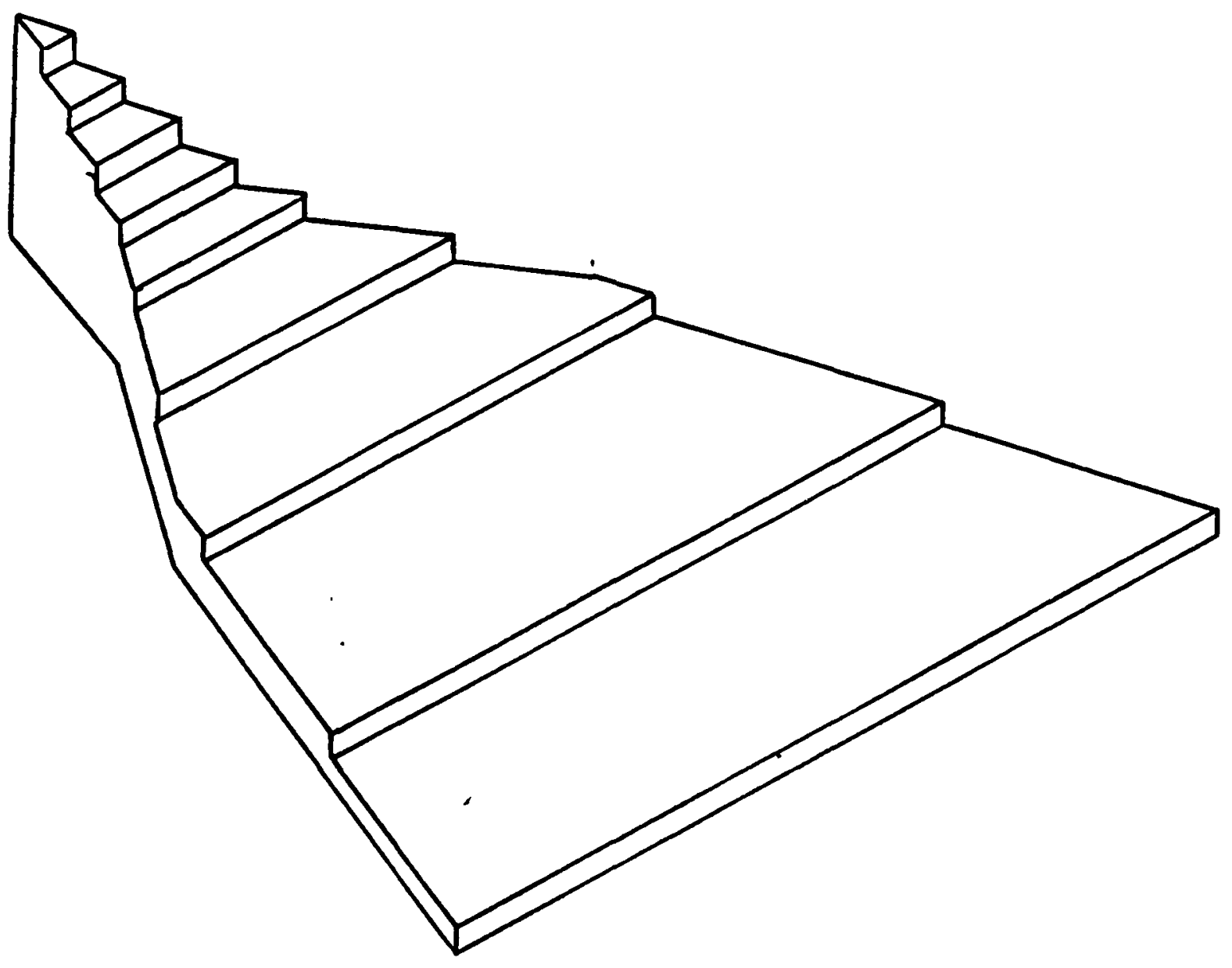

Figure A-7. Graphic View of the Uniform Dose Internal Model 


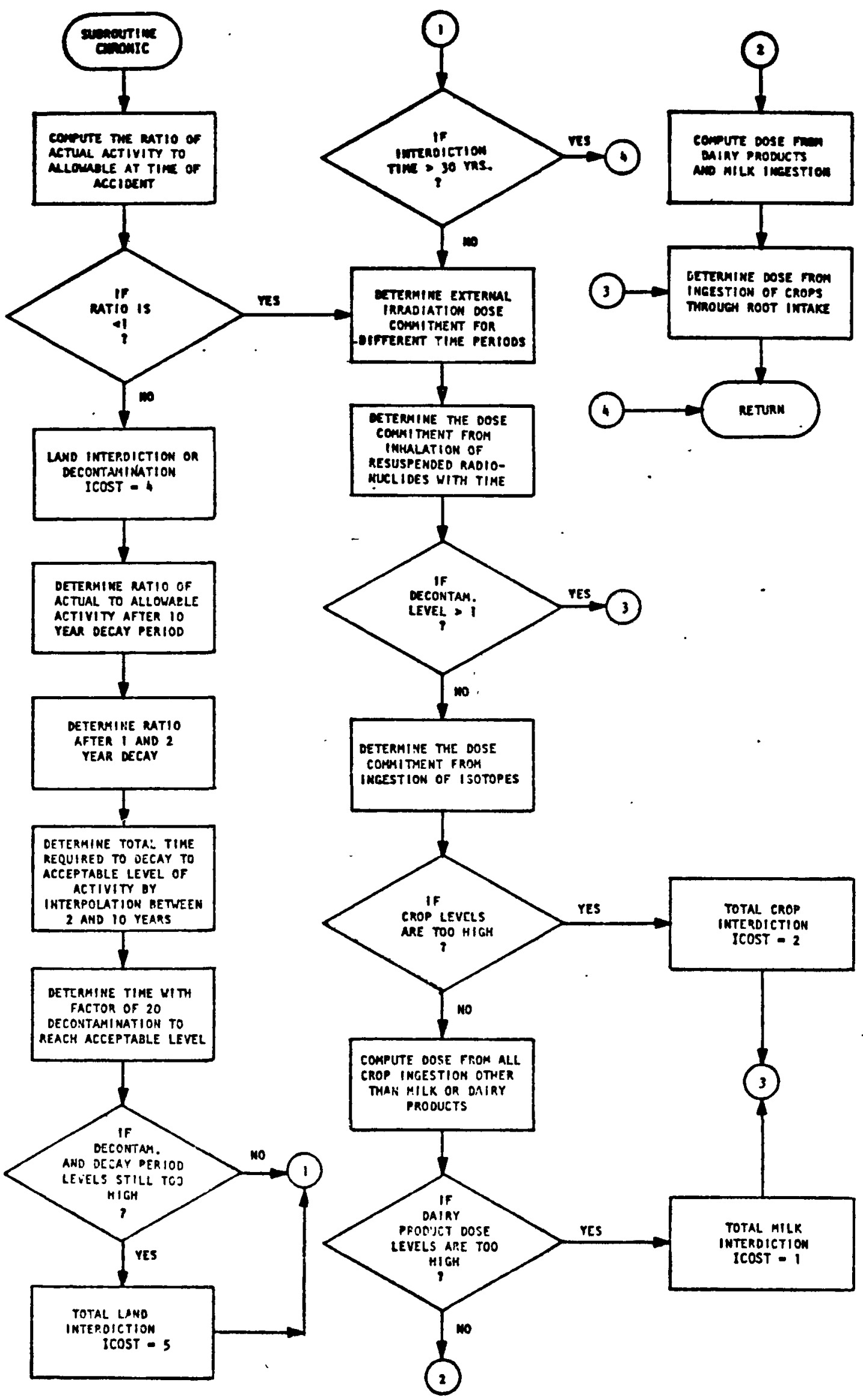

Figure A-8. Flow of CHRONX, the Chronic Effects Model 


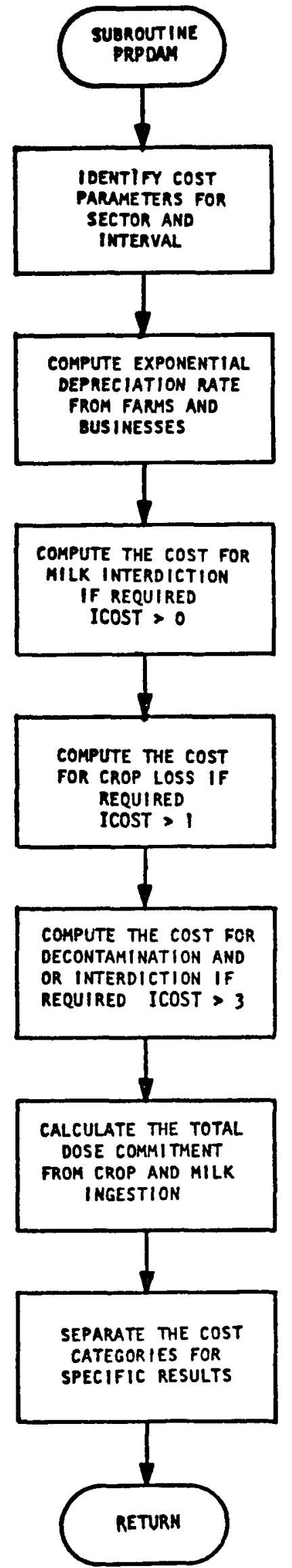

Figure A-9. Flow of PRPDAM, the Property Damage Model 
Appendix B. Quick Reference to the CRAC2 Models

This appendix contains a set of four tables that out ine the CRAC2 models described in section 3 . These tables serve as quick references to the models of the CRAC2 code. Table BI is a summary of these models in the same order as they are described in section 3. Table $B 2$ is a summary of the importance sampling method used to select meteorological sequences. Table B3 outlines the parameterization of the atmospheric dispersion model and the parameter values that are normally utilized in the model. Table B4 summarizes the dose-effect relationships that are used in the CRAC2 health effects models. 
Table Bl

Models Adopted for Use in the CRAC2 code

\section{Meteorological Sampling Schemes}

A. Random sompling:

Meteorological sequences are selected by $r$ andom choice of starting hours. The weight of each meteorological sequence 15 the reciprocal of the total number of sequences selected.

B. Stratified random sampling:

Meteorological sequences are selected by random choice with the starting hours stratified by day and night and by month. The welght of each meteorological seguence 15 the rect

c. Stratified sampling:

Meteorological sequences are selected cyclically with the starting hours occuring every four days of thirteen hours. The weight of each meteorological sequences selected.

D. Importance sampling (See Table B2):

Meteorological data are sorted into categories chosen to represent the years weather data and to assure representation of those weather sequences which can produce serious accident consequences. A number of meteorological sequences are selected randomly from eoch category using the Latin hypercube selection algorithm. The weight of each meteorological sequence is the ratio of the number of 1 tems in the category to the product of the number of 1 tems selected from the category and the total number of weather sequences.

11. Atmospheric D1spersion (See Table B3)

A. Dispersion model:

straight-1ine Gaussian top hat plume model that treats a single reflection from the ground and utilizes time varying meteorological data.
B. Plume rise model:

Empirical model described by Briggs

c. Plume depletion processes:

Wet and dry deposition and radionuclide decay

1. Dry deposition model:

Source depletion model utilizing a nucliae dependent, constant deposition velocity

2. Wet deposition model

Washout model ut 21121 ng a washout coefficient that is a function of rain rate and thermal stability

D. Buslding wake ef fects:

virtual source model

III. Dosimetric Models (See Table B4)

A. Short term exposure pathways:

1. Internal radiation due to inhalation of material (inhalation):

Dose models compatible with those of the ICRP-30

2. External r-radiation from the passing cloud (clouoshine):

Semi-infinite cloud model with finite cloud correction

3. External $r$-radiation dose from deposited material (groundshine): Smooth infinite plane approximation

B. Long term exposure pathways:

1. External 1-radiation from deposited material.

1) Smooth infinite plane approximation

11) Weathering of deposited material accounteo for by use of a time varying factor based on 
2. Inhalation of material resuspended from the ground. Dose models compatible with those of the ICRP-30.

3. Ingestion of contaminated food stuffs. Dose models of crop and milk ingestion pathways resulting from the direct deposition of $\mathrm{CS}$. Sr, and I onto crops and the root uptake of $\mathrm{Cs}$ and $\mathrm{Sr}$.

\section{Emergency Response}

A. Evacuation models:

1. Constant effective evacuation speed model utalized in the wash-1400 model

2. Constant evacuation speed model incorporating a delay time before public movement and detalled tracking of evacuees

\section{B. Sheltering model:}

A11 people living outside of the evacuation area and within the specifled sheltering radius are sheltered
using the applicable cloud and ground shielding tactors.

c. Relocation model:

All people living outside the evacuation area are relocation option desired.

1. People in the non-evacuating intervals will be relocated at 7 days following deposition. If. however, the 7-day external total bone marrow groundshine dose approaches lethal levels 1200 rewi), relocation will occur at 24 hours.

2. People in the non-evacuating intervals will be relocated at 24 hours following deposition.

3. People in the non-evacuating intervals will be relocated at the number of days following
deposition specified by the user of the model. v. Health Effects Models

A. Organs considered for health impacts:

1. Lungs

2. Total bone martow

3. Skeletal bone

4. Total endosteal cells

5. Stomach wall

6. Small intestines and contents

7. Upper large intestine wall

8. Lower large intestine wall

9. Thyroid

10. Other tissues; tissues other than lungs, bone marrow, walls of the gastrointegtinal tract, and thyroid

11. Whole body

12. Testes

13. Ovaries

B. Health effects normally considered:

1. Early death due to irradiation of:

1) Total bone marrow

11) Lungs

111) Lower large intestine wall

2. Early injuries by organ name and early effect:

2) Whole body - prodonal vomiting

i1) Lungs - lung fibrosis

11) Lower large intestine wall - lower large intestinal wall injury

iv) Thyroid - thryold damage 
3. Fatal cancers by organ name and latent effect.

1) rotal bone marrow - leukemia

11) Lungs - lung cancer

iii) Other tissues - breast cancer

(v) Skeletal bone - bone cancer v) Lower large intestine wall - gastrointestinal

v1) Other tissues - other cancers

4. Other latent effects by organ name: i) Thyroid - total thyrasd cancerous and
noncancerous nodules

11) Whole body - total latent whole body cancers

c. Dose effect relationship models:

1. Early effects:

Piecewise linear dose relationships with dose thresholds below which no health effects result

2. Lote effects:

The pattern of appearance of late effects from early intiation exposures takes into account aging of the population.

1) The central estimate model - utilizes dose effectiveness factors that reduce the effectiveness of low doses and low dose rates.

ii) Alternative Iınear, no threshold model known as the linear model.

\section{vi. Economic Effects Models}

A. Cost of early mitigative actions - evacuation:

The estimate of evacuation costs is based on the numbe of Individuals evacuated multiplied by the average cost per evacuee.

B. Costs of long term mitigative actions

These costs are dependent on the measures implemented in each spatial interval. Six mitigative measures and

1. Land area interdiction followed by land and property decontamination

2. Immediate decontamination and inhabitation of area

3. Milk and crop disposal

4. Crop disposal

5. Milk disposal

6. No long-term actions 
Table $B 2$

Importance Sampling of Meteorological Data

\section{Categorization hof Meteorological Data}

The weather sequences that comprise the meteorological dat are sorted into 29 weather sequence categories consisting egories, and 17 initial stability/windspeed categories.

\section{A. Rainfall categorses:}

Weather sequences in which rainfall begins within 30 miles of the reactor site are assigned to the rainfall categories

\section{Identifying numbers \\ of the rainfall}

$\begin{array}{lc}1 & 0 \\ 2 & 0-5 \\ 3 & 5-10 \\ 5 & 10-15 \\ 5 & 15-20 \\ 6 & 20-25 \\ 7 & 25-30\end{array}$

B. Windspeed slowdown categories:

Weather sequences which are not assigned to the rainfall categories in which there is an occurrence of - windspeed slowdown from greater than 3ms to less within 30 miles of the reactor site assigned to the windspeed slowdown categor 1 es.

Identifying numbers of the windspeed Distance (miles) from the slowdown an wandsped from greater than $3 \mathrm{ss}^{-1}$ to less than $2 \mathrm{~ms}$ -

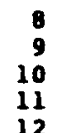

$0-10$
$10-15$
$15-20$
$20-25$
$25-30$

C. Initial stability/windspeed categories:

Weather sequences which are not assigned to the rainfall or windspeed slowdown categories are assigned to an initial stability/windspeed category in

accordance with the initial conditions of the beginning of the weather sequence.

I dent Ifying numbers of the initial stability Stability Initial windspeed categor ies

A, B, and C
A, B, and C
D
$D$
$D$
$D$
$D$
$D$
E
E
E
$E$
E
E
$F$
$F$
$F$
$F$
Windspeed
(ms-1)

13
14
15
16
17
18
19
20
21
22
23
24
25
26
27
28
29

I1. Number of Samples Selected

The user may specify the number of samples selected from each of the categories. For most applications the number of samples selected from each category is 4 .

III. Sampling Technique

The Latin hypercube sampling method is used to select samples from each of the weather sequence categories. 
Table 83

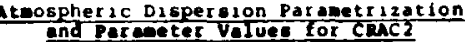

\section{Hor izontel Diepersion}

A. Stendard devietion of Gaussien pluse in the
borizontal direction, oy:

$$
o_{y}=o_{y_{P G}}\left(\frac{T}{0.05}\right)^{0}
$$

where ${ }^{1} Y G_{2}$ is the standard devilation obtasned from the Gur corresponding to : 3 anute release duration. ur corresponding to 3 mute release duration.
1s the relous duration in hours specified by the

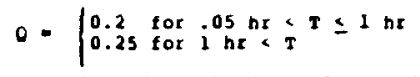

B. Representation of the horizontal Gaussian profilet The Gaussian proftle 18 approximated by a $82 n g l e$ etep
top hat. function of width $3 \sigma_{y}$ centered on the center line. The amplitude of the "top nat" is 0.03 of the Gauscien peak so that the area under the

c. Growth rates for oy: Grouth rates for oy in each patial interval are
eatimated from the valua in the previous ancerval

\section{Verticsl Dispersion}

A. Stenderd deviation of Gousian plune in the
horizontel direction. Oz:

$$
o_{z} \cdots o_{z_{p G}}\left(\frac{10}{3}\right)^{0.2}
$$

where $a_{z p g} 12$ the standard deviation abtained for the pastegil Gifford curves parameterized by mortin

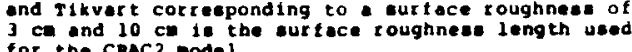

B. Expansion of $\mathrm{O}_{2}$ an influenced by the top of the The linst on the expansion of $o_{z}$ by the lop of the mixing layer is treated According to the
procedures of Turnet. For aluing leyer of depth $L$ :

1. Power law paraneterization of $\mathrm{O}_{2}$ for $\mathrm{O}_{2} \leq .465 \mathrm{~L}$.

2. Linear parameterization of $\mathrm{O}_{2}$ for $.465 \mathrm{~L} \times \mathrm{O}_{2}<$. BL

3. Uniforg vertical profile beyond the diatance at

c. Growth rater for $\mathrm{O}_{\mathbf{z}}$ :

Growth rates for $\mathrm{O}_{2}$ are estamated an each interval from the value of the previous interval

111. Mixing Heights

A. For atable conditions, lae. stability categorses
and p, the inversion layer io ground baned and no mixing depth is ossumed.

B. For unstable and neutral conditions, 1.e. stability ayer depth. are specified by the user (velues

IV. Plune Rise

A. Empiricol model of Briggs 18 utilized. The helght of

3. The helght of the plune rise 18 not iffected by the

depth of the mxing layer.

c. Wo lift off criterion is used.

\section{Plune Depletion}

A. Redionuclide decay:

Redionuclide decay with daughter bulldup

B. Dry deposition: Wucl ide dependent cource deplation nodel nolmally all nuclidee except for the noble gasee and a

c. Wet deposition:

Nuclide dependent washout model that 10 f function of

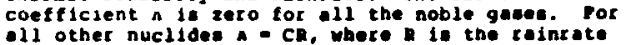
in $a$ he and

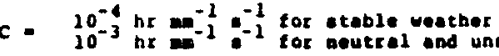

vi. Duilding Wake Eftecte

Virtual source model with initial values

$$
\begin{aligned}
& 0_{y}=\frac{1}{3} w \\
& 0_{8}=\frac{1}{2.15} \text { \# }
\end{aligned}
$$

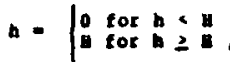

where wis the bulloling uldet, Is the bullding height ind $h$ is the height of releees 
Table 84

$\frac{\text { Dose-Effect Relationsh1ps Used in CRAC2 }}{\text { Hesith Effect5 ModeIB }}$

1. Early Death*

A. Total bone marrows

Mortallty incident to the bone arrow dose

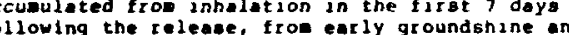
cloudehine, ind from one-half of the anhalation cose

$$
\begin{aligned}
& L D_{5}=105 \text { rad } \\
& L D_{50}=510 \mathrm{rad} \\
& L D_{95}=605 \text { rad }
\end{aligned}
$$

B. Lung

Mortality incident to the lung dose accumulated from Inhalation in the first year following the release

$$
\begin{aligned}
& \text { LD } 5: 7040 \mathrm{rad} \\
& \text { LD50: } 18800 \mathrm{rad} \\
& \text { LD95 } 23700 \mathrm{rad}
\end{aligned}
$$

c. Lover lorge intestinal well

Mortality Incident to the lower large intestinal wall following the release and frow oerly groundatine and cloudehinet

$$
\begin{aligned}
& \text { LDS }=2150 \text { rad } \\
& \text { LD50 }=3500 \text { red } \\
& \text { LDg5 } \\
& \hline 050 \text { rad }
\end{aligned}
$$

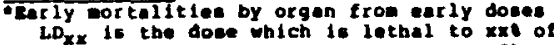
pox populetion. The LDix veluee
11. Early Injury**

a. Prodromel vamiting

Morbadity incrdent to the unole body dose acculaulated in the first 2 days following the release. from early groundsh at any rate zerger than $20 \mathrm{red}$ per days

$$
\begin{aligned}
& M D_{5}=71 \mathrm{rad} \\
& M D_{50}=202 \mathrm{rad} \\
& M D_{95}=348 \mathrm{rad}
\end{aligned}
$$

a. Lung fibrosis:

Morbidity incldent to the Lung dose accusulated f 70 om inhalation in the first year lollowing the re

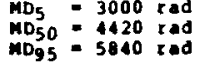

c. Lower lerge intestinal wall injury

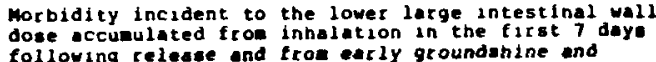
following rele

$$
\begin{aligned}
& M D_{5}=1000 \mathrm{rad} \\
& M D_{50}=11710 \mathrm{red} \\
& M D_{5}=2920 \mathrm{rad}
\end{aligned}
$$

MEmTy morbTdties from early doses

\begin{tabular}{|c|c|c|}
\hline Organ & Letent Cancer & 0-1 ys Risk coefficient \\
\hline telt Bone & 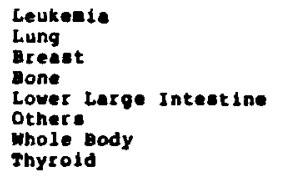 & 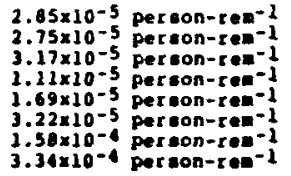 \\
\hline
\end{tabular}

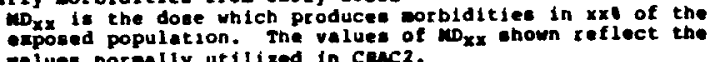

III. Hetel Cancers:

1. An ebeolute riak model is used in CRAC2 to model totel

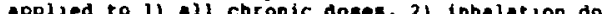
recelved within 1 your of tor the occident, 3) eorly
groundenine and cloudah ine doees to ootimate fetel

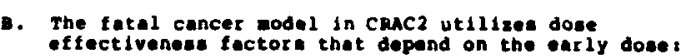
Ear Iy Dose Accuanulatod Dose Effectivenoss $30 \div 30 \mathrm{rex}$ .$::^{2}$

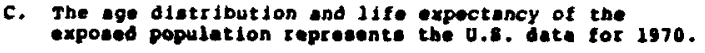

D. The fatel cancer modal reflecte latency period 
USNRC Distribution Contractor (CDSI)

7300 Pearl street

Bethesda, Maryland 20014

100 Copies for AN

$\begin{array}{ll}\text { USNRC } & \text { S. Acharya (10) } \\ \text { USNRC } & \text { R. Blond (25) }\end{array}$

USNRC J. Martin

Joseph C. S. Liu

Yankee Atomic Electric Company

1671 Worcester Road

Framingham, MA 01701

Dennis strenge

BPNL

Battelle Boulevard

Richland, WA 99352

Dave Simpson

W5C 178 C-K W10 C126C-K

Tennessee Valley Authority

400 Commerce Avenue

Knoxville. TN 37902

Charles Hoot

TO-E-289

General Atomic

P. O. Box 81608

San Diego, CA 92138

Dr. Fred Finlayson

Aerospace Corporation

P. O. Box 92957

Los Angeles, CA 90009

Harold A. Mitchell

Technology for Energy Inc.

10770 Dutchtown Road

Knoxville, TN 37922

Alex Kasprak

Stone and Webster Eng. Corp.

P.O. BOX 2325

Boston, MA 02107

Chris schamp

Babcock and wilcox

P. O. Box 1260

Lynchburg, VA 24505

Geoff Kaiser

NUS Corporation

910 Clopper Road

Gaithersburg. MD 20878

Dist-1 
Robert Haight

Radiological Programs

WPPSS

3000 George Washington Way

Richland, WA 99352

Paul Hsieh

Reliability Engineering

Bechtel Power Corporation

777 East Eisenhower Parkway

Ann Arbor, MI 48104

David Prezbindowski

WPPSS

3000 George Washington Way

Richland, WA 99352

Dr. Paul J. Wood

Wood, Leaver and Associates

P. O. Box 276

Monroeville, PA 15146

Johnny Collins

University Computing Company

$1930 \mathrm{Hi}$ Line Drive

Dallas, TX 75207

R. O. Gumprecht

Sargent and Lundy Engineers

55 East Monroe street

Chicago, IL 60603

Tim Lee-Thorp

EDS Nuclear

220 Montgomery street

San Francisco, CA 94104

Frank S. Dombeck

CYGNA Energy Services

225 Stevens street, Suite 110

Solano Beach, CA 92075

Duane Stevenson

Science Applications, Inc.

5 Palo Alto Square

Suite 200

Palo Alto, CA 94304

Richard N. Swanson

Nuclear Engineering

Boston Edison Company

800 Boylston street

Boston, MA 02199 
Michael C. Cheok

NUS Corporation

910 Clopper Road

Gaithersburg, MD 20878

Dr. William Nixon

UKAEA SRD

Wigshaw Lane

Culcheth

Warrington WA3 $4 \mathrm{NE}$

Cheshire

ENGLAND

David Yue

ORNL

P. O. Box Y

Building 9104-1

Oak Ridge, TN 37830

H. A. Careway

Nuc. Energy Bus. Op., M/C 769

General Electric

175 Curtner Avenue

San Jose, CA 95125

Dr. M. Moghari

Nuclear Associates International

6003 Executive Boulevard

Rockville, MD 20852

Scott Matthews, TSB

EG\&G Idaho

P. O. Box 1625

Idaho Falls, ID 83415

Chuck Johnson

S. Levy Inc.

Suite 725

1999 South Bascom Avenue

Campbell, CA 95008

Keith Woodard

Pickard, Lowe and Garrick, Inc.

120018 th street NW

washington, D.C. 20036

David G. Vreeland, L-140

University of California

P. O. Box 808

Livermore, CA 94550

Mr. U1f Tveten

Institute for Energiteknikk

Postboks 40

N-2007 Kjeller

NORWAY 


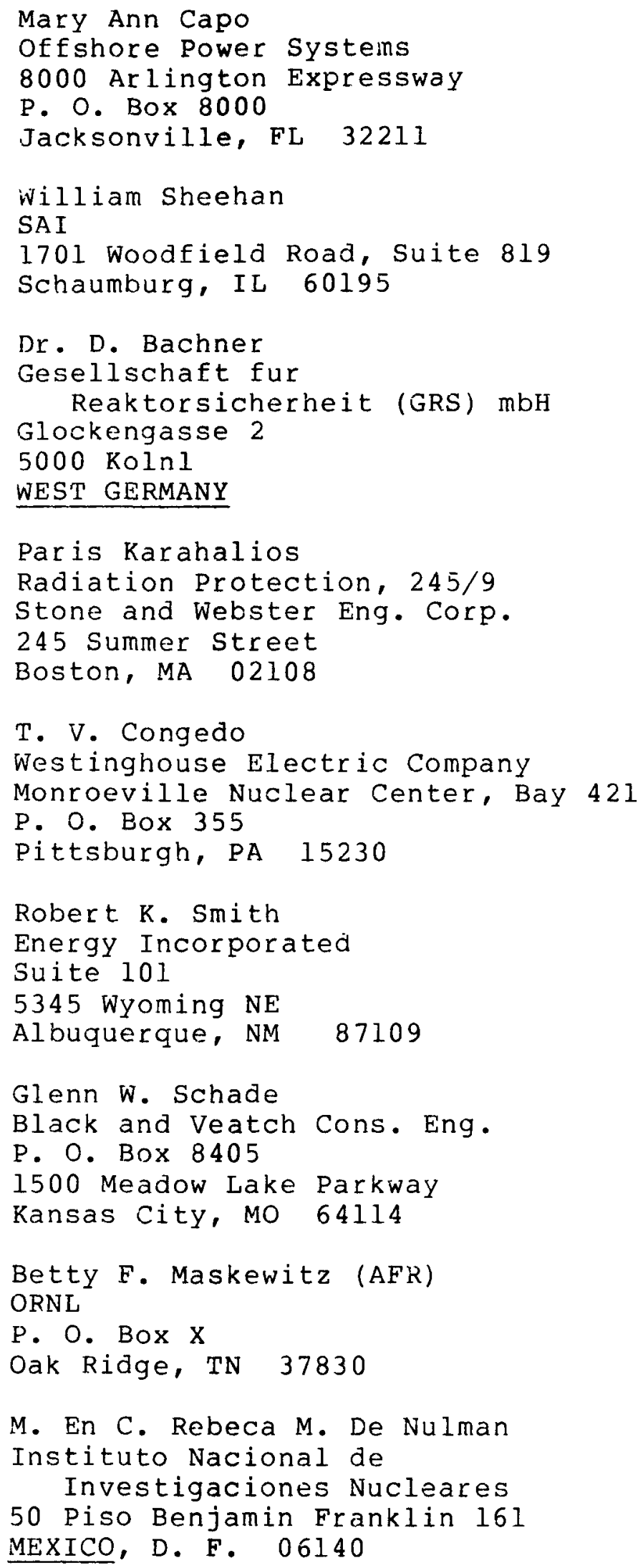


James A. Stoddard

Division 015

Science Applications, Inc.

1200 Prospect Street

P. O. Box 2351

La Jolla, CA 92038

Arthur Tingle, 130

BNL

Upton, NY 11973

Mr. Jorge Schulz

Bechtel Power Corporation

Fifty Beale Street

P. O. Box 3965

San Francisco, CA 94119

CFE-PNLV

Ingenieria Ambiental

At'n: FIS. Javier Alvarez-Gasca

Apartado Postal Num. 53

Cardel, Ver.

MEXICO

Ms. Julie M. Pietrzak

National Energy Software Center

Argonne National Laboratory

9700 South Cass Avenue

Argonne, IL 60439

Dennis R. Damon

Reactor Safety, Mail Code s-23

General Electric Company

310 Deguigne Drive

P. O. Box 508

Sunnyvale, CA 94086

Randall C. Foltz

Nuclear Systems Manager

United Computing Systems, Inc.

Suite 106

7700 Leesburg Pike

Falls Church, VA 22043

Mr. Fernando Legarda

El Profesor de las Escuela Tecnica

Superior de Ingenieros Indust.

Departamento de Fisica Nuclear

"L. JOSE DE TORRONTEGUI"

Bilbao

SPAIN

Soren Thykier-Nielsen

Health Physics Department

Riso National Laboratory

Postbox 49

DK-4000 Roskilde

DENMARK 
John Bartzis

Greek Atomic Energy Commission

Aghia Paraskevi Attikis

GREECE

T. Iijima

Division of Reactor Safety Eval.

Tokai Research Establishment

Japan Atomic Energy Res. Inst.

Tokai-mura

Ibaraki-ken 911-13

JAPAN

Vincent J. Cordo, Sr.

Envirosphere Company

160 Chubb Avenue

Lyndhurst, NJ 07071

William Groot

HMM Associates

255 Bear Hill Road

Waltham, MA 02154

Rod Buckles

NUS Corporation

Licensing Information Service

2536 Countryside BIvd.

Clearwater, FL 33515

Dr. E. Stern

Head, Risk Assessment Dept.

Atomic Energy Commission

Licensing Division

P. O. Box 7061

Tel-Aviv

ISRAEL

Roger Breeding

Energy Inc.

P. O. box 736

Idaho Falls, ID 83402

Steve Masciulli

PASNY

123 Main street

White Plains, NY 10601

Dr. Mohan Thadani

Mail Stop P-802

USNRC

Washington, DC 20555

Peter Konichek

Boeing Computer Services

565 Andover Park West

Tukwila, WA 98188

Dist-6 
Steve Gibbon, N2-1

Philadelphia Electric

2301 Market Street

Philadelphia, PA 19101

S. M. Mondro

EG\&G Idaho, Inc.

P. O. Box 1625

Idaho Falls, ID 83415

Jong Sook Hong

Korea Advanced Energy Research Inst.

Daeduk Engineering Center

P. O. Box 7

Daeduk Sanji, Chung-nam

KOREA

Dr. F. J. Mogolesko

Boston Edison Company

25 Braintree Hill Office Park

Barintree, MA 02185

Sebastiano Serra

Direzione delle Costruzioni (DCO)

Ente Nazionale per L'energia Electrica

Via G. B. Martini, 3

Casella Postale N. 386

I-00100 Roma, ITALY

Ms. Carla Brofferio

DISP/ARA/SCA

Comitato Nazionale per L'energia Nucleare

125 Viale Regina Margherita

00198

Roma, ITALY

J. L. Elliot

Safety Review, Analysis, and Licensing Div.

Duke Power Company

P. O. Box 33189

Charlotte, NC 28242

Dr. Douglas G. Smith

Air Quality Studies Division

Environmental Research and 'r'echnology, Inc.

696 Virginia Road

Concord, MA 01742

Bill Rhyne

H\&R Technical Associates

P. O. Box 215

Oak Ridge, TN 37830 

6400 A. W. Snyder
6410 J. W. Hickman
6411 A. S. Benjamin
6414 D. M. Ericson. Jr.
6415 D. C. Aldrich (3)
6415 D. J. Alpert (3)
6415 D. E. Bennett
6415 R. P. Burke (3)
6415 J. M. Griesmeyer
6415 R. L. Iman
6415 C. D. Leigh
6415 R. M. Ostmeyer
6415 L. T. Ritchie (30)
6415 J. L. Sprung
6415 D. R. Strip
6415 A. R. Taig
6415 J. D. Johnson (3)
6432 K. G. Adams
$6420 \mathrm{~J} . \mathrm{V}$. Walker
6430 N. R. Ortiz
6440 D. A. Dahlgren
8214 M. A. Pound
3141 C. M. Ostrander(5)
3151 W. J. Garner 